\title{
Arnol'd tongues for a resonant injection-locked frequency divider: analytical and numerical results
}

\author{
M. V. Bartuccelli ${ }^{1}$, J. H.B. Deane ${ }^{1}$, G. Gentile ${ }^{2}$, F. Schilder ${ }^{1}$ \\ ${ }^{1}$ Department of Mathematics, University of Surrey, Guildford, GU2 7XH, UK. \\ E-mails: m.bartuccelli@surrey.ac.uk, j.deane@surrey.ac.uk, f.schilder@surrey.ac.uk \\ ${ }^{2}$ Dipartimento di Matematica, Università di Roma Tre, Roma, 00146, Italy. \\ E-mail: gentile@mat.uniroma3.it
}

\begin{abstract}
In this paper we consider a resonant injection-locked frequency divider which is of interest in electronics, and we investigate the frequency locking phenomenon when varying the amplitude and frequency of the injected signal. We study both analytically and numerically the structure of the Arnol'd tongues in the frequency-amplitude plane. In particular, we provide exact analytical formulae for the widths of the tongues, which correspond to the plateaux of the devil's staircase picture. The results account for numerical and experimental findings presented in the literature for special driving terms and, additionally, extend the analysis to a more general setting.
\end{abstract}

\section{Introduction}

The locking of oscillators onto subharmonics of the driving frequency (known as frequency locking or frequency demultiplication) has been well known in electronics since the work of van der Pol and van der Mark [22]; see also [12. In the frequency-amplitude plane, locking occurs in distorted wedge-shaped regions (Arnol'd tongues) with apices corresponding to rational values on the (scaled) frequency axis. If one plots the ratio of the driving frequency to the output frequency versus the driving frequency, one obtains a so-called devil's staircase, i.e. a self-similar fractal object, where the qualitative structure is replicated at higher levels of resolution, with plateaux corresponding to rational values of the ratio.

The frequency locking phenomenon, the existence of the Arnol'd tongues, and the devil's staircase structure have been proved rigorously in some mathematical models, such as the circle map [3], and studied numerically or experimentally for several electronic circuits, such as the van der Pol equation [9, 17, the Josephson junction [1, 13, 20, the Chua circuit [18, among others.

In this paper we are interested in studying both analytically and numerically an electronic circuit, namely a resonant injection-locked frequency divider (ILFD), first considered in [15]. In [16] a differential equation was introduced to describe the circuit and it was shown that the numerical integration of the equation reliably reproduces the experimental data.

In [4, the differential equation describing the ILFD was studied with the purpose of explaining analytically the appearance of frequency locking. In particular, the full differential equation in question was shown to be of the form $u^{\prime \prime}+u^{\prime} h(u)+k(u)+\mu \Psi\left(u, u^{\prime}, t\right)=0$, where $h(u)$ and $k(u)$ are even and odd functions of $u$, respectively, the prime denotes differentiation with respect to $t$, and $\mu$ is the amplitude of the perturbation $\Psi$, which is taken to be periodic in $t$, with frequency $\omega$, i.e. $\Psi\left(u, u^{\prime}, t\right)=\Psi\left(u, u^{\prime}, t+2 \pi / \omega\right)$. If the drive is purely sinusoidal, as in [16], the Fourier series 
expansion of $\Psi\left(u, u^{\prime}, t\right)$,

$$
\Psi\left(u, u^{\prime}, t\right)=\sum_{\nu \in \mathbb{Z}} \mathrm{e}^{\mathrm{i} \nu \omega t} \Psi_{\nu}\left(u, u^{\prime}\right),
$$

contains only the first harmonics $\nu= \pm 1$ (i.e. $\Psi_{\nu}\left(u, u^{\prime}\right)=0$ for $|\nu|>1$ ).

When $\mu=0$, the system is unperturbed, and the differential equation is of a particular form known as the Liénard equation [10, 9]; the best known example of this type is the van der Pol equation. Under suitable assumptions on $h$ and $k$, the Liénard equation admits a globally-attracting limit cycle.

The phenomenon of frequency locking manifests itself in the ILFD when the ratio of the driving frequency $\omega$ to the output frequency $\Omega$ is plotted against $\omega$. When $\omega$ is close to a rational multiple $\rho=p / q$ of the frequency $\Omega_{0}$ of the limit cycle of the unperturbed system, then $\Omega$ is fixed such that $\omega=\rho \Omega$. Therefore the plot has a devil's staircase structure [15, with plateaux corresponding to rational values of the ratio $\omega / \Omega$. If $\omega / \Omega=p / q$ one says that there is a resonance (or synchronisation) of order $p: q$. For purely sinusoidal perturbations $\Psi$, such as those considered explicitly in [15, 16, the main plateaux correspond to even values of $\rho$ (a physical argument was given in [23]). The perturbation theory approach taken in [4] successfully explains the experimental observations, by computing quantitatively the way in which the widths of the plateaux depend on the amplitude of the perturbation $\mu$, assumed small.

In an alternative visualisation of the situation, a two-dimensional plot showing where locking takes place is constructed in the $(\omega, \mu)$ plane. The Arnol'd tongues have widths and centre-lines which vary as some (explicitly computable) integer power of $\mu[4$. The experimentally-observed dominance of tongues for which the ratio $\omega / \Omega_{0}$ is close to an even integer can be explained by the fact that only these tongues have a width of order $\mu$ : all other tongues grow in width as some higher power of $\mu$. Specifically, if $\rho \in \mathbb{Q}$ and $\Delta \omega(\rho)=\{\omega: \omega / \Omega=\rho\}$ is the width of the corresponding locking interval at fixed $\mu$, it was proved that, for sinusoidal perturbations,

$$
\Delta \omega(2 n / k)=O\left(\mu^{k}\right), \quad \Delta \omega((2 n-1) / k)=O\left(\mu^{2 k}\right)
$$

for all $k, n \in \mathbb{N}$ such that $2 n / k$ and $(2 n+1) / k$ are irreducible fractions. The centre-lines are vertical for $\rho=2 n$ and bend away from the vertical by a distance of order $\mu^{2}$ for all other values of $\rho$. In [4] we also stressed that the property (1.1) strongly depends on the particular form of the drive, more precisely on the fact that, as in [15, 16, the driving was taken to contain only the first harmonics.

More generally, one can express $\Delta \omega(\rho)$ as a power series (perturbation series) in $\mu$,

$$
\Delta \omega(\rho)=\sum_{k=1}^{\infty} \mu^{k} \Delta_{k} \omega(\rho) .
$$

If $k_{0} \in \mathbb{N}$ is the first integer such that $\Delta \omega_{k_{0}}(\rho) \neq 0$ then from (1.2) one obtains $\Delta \omega(\rho)=$ $\mu^{k_{0}} \Delta_{k_{0}} \omega(\rho)+O\left(\mu^{k_{0}+1}\right)$.

The convergence of the perturbation series for $\mu$ small enough — yielding analyticity in $\mu$ in a neighbourhood of the origin — was discussed and proved in [4]; see also [8]. Hence, by keeping only the lowest order terms means that in (1.1) we are looking at the leading contributions, without making any uncontrolled truncation. The coefficients $\Delta_{k} \omega(\rho)$ are given in the form of suitable integrals. However, it is not possible to reduce this computation to the integration of elementary functions, because the integrands involve functions which are known only numerically. Thus, the computation of the integrals requires some work, which we also discuss in this paper.

A first order analysis of the locking intervals (in the same spirit as in [4) is also performed in [2, where only sinusoidal perturbations are considered; in particular no prediction is made for resonances $p: q$ with $p / q \notin 2 \mathbb{N}$, as this would require a higher order analysis. More general perturbations are considered in [14, where a different approach is followed. However, this involves 
approximations which, ultimately, correspond to a first order analysis. By contrast, the analysis performed in [8] and further developed here allows us to go to arbitrary perturbation orders, with a control on the remainder. Thus, not only one can find an exact analytical expression for the leading order of the locking interval of any resonance, but in principle one can also compute any locking interval within any desired accuracy. In [7, the lack of accurate analytical methods to predict the locking range was deplored: in our opinion our analysis, which makes no approximation, fills this gap. Of course, for practical purposes, the computation of the locking interval for any given resonance requires solving numerically some integrals (which become increasingly complicated as the perturbation order increases). It would be desirable to have a formula for the locking interval in terms of the parameters $\alpha$ and $\beta$ of the system, were one to exist; we point out that in [2] an asymptotic formula is given in the limit of $\alpha=\infty$ and $\beta$ large.

In further detail, the motivation for the current paper, which completes the analysis of [4] and also concentrates on numerics related to the ILFD problem, is as follows:

1. To compute the coefficients of the powers of $\mu$ explicitly, at least for the lowest perturbation orders, so as to give a quantitative expression for the width of the tongues, for more general perturbations than those considered in [4].

2. To investigate numerically how large $\mu$ can be for the analysis, which is carried out under the assumption that $\mu \ll 1$, to break down.

3. To compute numerically the Arnol'd tongue diagram in the $(\omega, \mu)$ plane in the case that the periodic part of the perturbation contains only one frequency, $\omega$. This allows us to obtain information for values of $\mu$ where perturbation theory does not apply. On the other hand, for smaller values of $\mu$, the analytical results provide a check on the reliability of the numerical analysis.

4. The same as 3 but in the case that the perturbation contains all integer multiples of $\omega$ : it was argued in [4] that the width of all tongues would then be proportional to $\mu$ and all the centre lines would be vertical. In particular we want to determine the constant of proportionality, i.e. the coefficient $\Delta_{1} \omega(\rho)$ in (1.2), and show that the higher the values of $p$ and $q$ in $\rho=p / q$, the lower the constant.

The rest of the paper is organised as follows. In Section 2 we summarise definitions and lemmas from 4 which are needed in the remainder of the paper, and extend the analysis to more general analytical driving, possibly containing all harmonics. In Section 3 we concentrate on analytical results concerning the Arnol'd tongues, by gathering together all information which can be obtained to any order of perturbation theory. In Section 4 we describe the algorithms used to carry out the computations of the integrals appearing in the theory. In Section 5 we give and discuss the numerical results: after checking that they agree with the theory where the latter applies (small $\mu$ ), we investigate how large $\mu$ can be for the theoretical predictions to be reliably used. Finally in Section 6 we draw some conclusions.

\section{Preliminary analytical results}

We recall the results of [4, and extend them to more general perturbations. Numbered lemmas which we refer to in this paper are taken directly from [4, and all proofs are given there too. Reference to [4] is given only for proofs and technical details, the discussion below being quite self-contained.

The system of ordinary differential equations that describes the ILFD can be put into the form

$$
u^{\prime \prime}+u^{\prime} h(u)+k(u)+\mu \Psi\left(u, u^{\prime}, t\right)=0,
$$


with

$$
h(u):=1-\beta+3 \beta u^{2}, \quad k(u):=u\left(\alpha-\beta+\beta u^{2}\right),
$$

and

$$
\Psi\left(u, u^{\prime}, t\right):=u^{\prime}\left(3 u^{2}-1\right) f(\omega t)+u\left(u^{2}-1\right)\left(f(\omega t)+\omega f^{\prime}(\omega t)\right),
$$

where here and henceforth $f^{\prime}$ denotes the derivative of $f$ with respect to its argument. The case $f(t)=\sin t$ (and hence $f^{\prime}(t)=\cos t$ ) was explicitly considered in 44. More generally one can consider any analytic $2 \pi$-periodic function

$$
f(t)=\sum_{\nu=1}^{\infty} \hat{f}_{\nu} \sin \nu t, \quad\left|\hat{f}_{\nu}\right| \leq \Phi \mathrm{e}^{-\xi|\nu|},
$$

where the bound on the Fourier coefficients $\hat{f}_{\nu}$ - for suitable positive constants $\Phi$ and $\xi$ follows from the analyticity assumption on $f$. For simplicity we confine ourselves to odd functions: considering functions whose Fourier expansion contains also cosines would overwhelm the analysis without shedding further light on the results.

For $\mu=0$, 2.1) reduces to the Liénard equation [6, 10]

$$
u^{\prime \prime}+u^{\prime} h(u)+k(u)=0,
$$

which we refer to as the 'unperturbed equation'. In order for it to have a globally-attracting limit cycle encircling the origin [10, 24] we require that $\alpha>\beta>1$ (this corresponds to the region of the parameter plane called design area in [7]). In that case, we designate $u_{0}(t)$ the solution to (2.5) corresponding to the limit cycle. Let $T_{0}$ be the period of $u_{0}(t)$ and let $\Omega_{0}=2 \pi / T_{0}$ be the corresponding frequency: $\Omega_{0}$ depends solely on $\alpha$ and $\beta$.

The unperturbed equation is autonomous, hence it clearly has the property that if $u_{0}(t)$ is a solution, then so is $u_{0}(t+T)$ for any constant $T$. Consequently, we can fix the origin of time so that $u_{0}(0)=U_{0}>0$ and $u_{0}^{\prime}(0)=0$. This has the effect of shifting the third argument of $\Psi$ by some time $t_{0}$, so $\Psi\left(u, u^{\prime}, t\right)$ becomes $\Psi\left(u, u^{\prime}, t+t_{0}\right)$ in (2.1).

We also note that the symmetry properties of $h(u)$ and $k(u)$ guarantee that $u_{0}(t)$ has the property

$$
u_{0}\left(t+T_{0} / 2\right)=-u_{0}(t) \quad \forall t \in \mathbb{R}
$$

which in turn yields that the Fourier expansion of $u_{0}(t)$ contains only odd harmonics (lemma 2.1).

It is convenient to rescale time by defining $\tau=\omega t$ so that $\Psi$ now has period $2 \pi$ in its third argument. After rescaling, the differential equation becomes

$$
\ddot{u}+\frac{1}{\omega} \dot{u} h(u)+\frac{1}{\omega^{2}} k(u)+\mu \bar{\Psi}\left(u, \dot{u}, \tau+\tau_{0}\right)=0,
$$

where a dot denotes differentiation with respect to $\tau, \tau_{0}=\omega t_{0}$, and we have defined

$$
\bar{\Psi}(u, \dot{u}, \tau)=\frac{1}{\omega^{2}}\left[\omega \dot{u}\left(3 u^{2}-1\right) f(\tau)+u\left(u^{2}-1\right)\left(f(\tau)+\omega f^{\prime}(\tau)\right)\right] .
$$

We have shown in [4 that if $\omega$ is 'close' to $p \Omega_{0} / q$, where $p, q \in \mathbb{N}$ are relatively prime, then the frequency $\Omega$ of the solution exactly equals $q \omega / p$ : the system is said to be locked into the $p: q$ resonance. How close $\omega$ has to be to $p \Omega_{0} / q$ depends on $\mu$ and on the resonance itself - quantitative investigation of this 'closeness' is the aim of the present paper.

Let $\rho=p / q \in \mathbb{Q}$. For $\omega$ close to $\rho \Omega_{0}$ put

$$
\frac{1}{\omega}=\frac{1}{\rho \Omega_{0}}+\varepsilon\left(\mu, \tau_{0}\right), \quad \text { where } \quad \varepsilon\left(\mu, \tau_{0}\right)=\sum_{k=1}^{\infty} \mu^{k} \varepsilon_{k}\left(\tau_{0}\right) .
$$


Unlike [4, for the sake of convenience, here we make explicit the dependence of $\varepsilon$ on $\tau_{0}$. The perturbation calculation is then carried out by substituting the expression (2.9) for $\omega$ in (2.7) and expanding in powers of $\mu$. This results in

$$
H(u, \dot{u}, \ddot{u}, \mu):=H_{0}(u, \dot{u}, \ddot{u})+\sum_{k=1}^{\infty} \mu^{k} H_{k}\left(u, \dot{u}, \tau+\tau_{0}\right)=0,
$$

where

$$
\begin{aligned}
H_{0}(u, \dot{u}, \ddot{u}) & =\ddot{u}+\frac{\dot{u} h(u)}{\rho \Omega_{0}}+\frac{k(u)}{\rho^{2} \Omega_{0}^{2}}, \\
H_{1}(u, \dot{u}, \tau) & =\varepsilon_{1}\left(\tau_{0}\right)\left(\dot{u} h(u)+\frac{2 k(u)}{\rho \Omega_{0}}\right)+\frac{\dot{u}\left(3 u^{2}-1\right)}{\rho \Omega_{0}} f(\tau)+u\left(u^{2}-1\right)\left(\frac{f(\tau)}{\rho^{2} \Omega_{0}^{2}}+\frac{f^{\prime}(\tau)}{\rho \Omega_{0}}\right), \\
H_{k}(u, \dot{u}, \tau) & =\varepsilon_{k}\left(\tau_{0}\right)\left(\dot{u} h(u)+\frac{2 k(u)}{\rho \Omega_{0}}\right)+\sum_{k_{1}+k_{2}=k} \varepsilon_{k_{1}}\left(\tau_{0}\right) \varepsilon_{k_{2}}\left(\tau_{0}\right) k(u) \\
& +\varepsilon_{k-1}\left(\tau_{0}\right)\left[\dot{u}\left(3 u^{2}-1\right) f(\tau)+u\left(u^{2}-1\right)\left(\frac{2 f(\tau)}{\rho \Omega_{0}}+f^{\prime}(\tau)\right)\right] \\
& +\sum_{k_{1}+k_{2}=k-1} \varepsilon_{k_{1}}\left(\tau_{0}\right) \varepsilon_{k_{2}}\left(\tau_{0}\right) u\left(u^{2}-1\right) f(\tau), \quad k \geq 2,
\end{aligned}
$$

where the last line of (2.11c) is missing for $k=2$.

In order to carry out the perturbation calculation to first order, we first write the unperturbed system in the form

$$
\dot{u}=v, \quad \dot{v}=-\frac{v h(u)}{\rho \Omega_{0}}-\frac{k(u)}{\rho^{2} \Omega_{0}^{2}} \equiv G(u, v)
$$

which has a unique $2 \pi \rho$-periodic solution $\left(u_{0}(\tau), v_{0}(\tau)\right)$ such that $v_{0}(0)=0$. The Wronskian matrix of equation (2.12) is

$$
W(\tau)=\left(\begin{array}{ll}
w_{11}(\tau) & w_{12}(\tau) \\
\dot{w}_{11}(\tau) & \dot{w}_{12}(\tau)
\end{array}\right)
$$

and satisfies

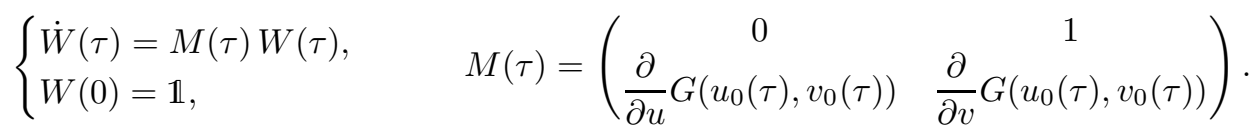

Lemma 4.1 then states that a solution to equation (2.14) is obtained by setting

$$
w_{12}(\tau):=c_{2} \dot{u}_{0}(\tau), \quad w_{11}(\tau):=c_{1} \dot{u}_{0}(\tau) \int_{\bar{\tau}}^{\tau} \mathrm{d} \tau^{\prime} \frac{\mathrm{e}^{-F\left(\tau^{\prime}\right)}}{\dot{u}_{0}^{2}\left(\tau^{\prime}\right)}
$$

where $F(\tau)$ is given by

$$
F(\tau):=\frac{1}{\rho \Omega_{0}} \int_{0}^{\tau} \mathrm{d} \tau^{\prime} h\left(u_{0}\left(\tau^{\prime}\right)\right)
$$

the constant $\bar{\tau} \in(0, \pi \rho)$ is chosen so that $\dot{w}_{11}(0)=0$, while the constants $c_{1}$ and $c_{2}$ are such that $W(0)=\mathbb{1}$ - it is shown in [4 that this choice can always be made.

By defining $r_{1}:=\ddot{u}_{0}(0)$, as in [4], and substituting this into (2.12), we find that

$$
r_{1}=-\frac{U_{0}\left(\alpha-\beta+\beta U_{0}^{2}\right)}{\rho^{2} \Omega_{0}^{2}} .
$$

By Remark 4.2 in [4], we have, additionally, that $c_{1}=-r_{1}$ and $c_{2}=1 / r_{1}$. 
We further define $f_{0}$ by $\rho \Omega_{0} f_{0}=\langle h\rangle$, the mean value of $h\left(u_{0}(\tau)\right)$, so that $f_{0}=F(2 \pi \rho) /(2 \pi \rho)$, and we write $F(\tau)=f_{0} \tau+\tilde{F}(\tau)$, where $\tilde{F}(\tau)$ is a $2 \pi \rho$-periodic function with zero mean. By lemma 1.2 one has $f_{0}>0$ (cf. also [6]).

Lemma 4.4 then states that there exist two $2 \pi \rho$-periodic functions $a(\tau)$ and $b(\tau)$ such that

$$
w_{11}(\tau)=a(\tau)+\mathrm{e}^{-f_{0} \tau} b(\tau), \quad w_{12}(\tau)=c a(\tau),
$$

for a suitable constant $c$. In order to develop perturbation theory for a $2 \pi p$-periodic solution, with $p \in \mathbb{N}$, which continues the unperturbed solution when $\mu \neq 0$, one writes

$$
u(\tau)=u_{0}(\tau)+\sum_{k=1}^{\infty} \mu^{k} u_{k}(\tau)
$$

where $u_{0}(\tau)$ has period $2 \pi \rho$ (and hence frequency $1 / \rho$ ). We have shown in 4 that there exist $2 \pi p$-periodic functions $u_{k}(\tau)$ such that the perturbation series (2.19) converges for $\mu$ small enough. The functions $u_{k}(\tau)$ are recursively defined (see equation (7.2) of [4) as

$$
u_{k}(\tau)=w_{11}(\tau) \bar{u}_{k}+w_{12}(\tau) \bar{v}_{k}+\int_{0}^{\tau} \mathrm{d} \tau^{\prime} \mathrm{e}^{F\left(\tau^{\prime}\right)}\left[w_{12}(\tau) w_{11}\left(\tau^{\prime}\right)-w_{11}(\tau) w_{12}\left(\tau^{\prime}\right)\right] \Psi_{k}(\tau),
$$

with

$$
\Psi_{k}(\tau):=\left[-\sum_{k^{\prime}=1}^{k} \mu^{k^{\prime}} H_{k^{\prime}}\left(u(\tau), \dot{u}(\tau), \tau+\tau_{0}\right)+G_{2}(u(\tau), \dot{u}(\tau))\right]_{k},
$$

where

$$
\begin{aligned}
G_{2}(u, v) & :=G(u, v)-G\left(u_{0}(\tau), v_{0}(\tau)\right) \\
& \left.-\left(u-u_{0}(\tau)\right) \frac{\partial}{\partial u} G\left(u_{0}(\tau), v_{0}(\tau)\right)-\left(v-v_{0}(\tau)\right)\right) \frac{\partial}{\partial v} G\left(u_{0}(\tau), v_{0}(\tau)\right)
\end{aligned}
$$

and the notations $[\cdot]_{k}$ means that we expand $u(\tau)$ and $\dot{u}(\tau)$ according to (2.19) and, after taking the Taylor series of the functions $H_{k^{\prime}}, k^{\prime}=1, \ldots, k$, and $G_{2}$, we keep the coefficients of all contributions proportional to $\mu^{k}$. In (2.20), the initial conditions $\bar{u}_{k}$ must be suitably fixed (again we refer to [4] for details), whereas $\bar{v}_{k}$ can be set equal to zero (cf. remark 5.1 of [4).

Considering first order in $\mu$, we obtain the first order compatibility condition that has to be satisfied if $u_{1}(\tau)$ is to be periodic, i.e. $\left\langle\mathrm{e}^{\tilde{F}} b \Psi_{1}\right\rangle=0$, where $\Psi_{1}(\tau)=-H_{1}\left(u_{0}(\tau), v_{0}(\tau), \tau+\tau_{0}\right)$. Expanding $f(\tau)$ according to (2.4) and using (2.11b), this gives

$$
\varepsilon_{1}\left(\tau_{0}\right) A+\sum_{\nu=1}^{\infty} \hat{f}_{\nu} \sum_{j=1}^{3}\left[B_{j 1 \nu} \cos \nu \tau_{0}+B_{j 2 \nu} \sin \nu \tau_{0}\right]=0,
$$

where

$$
A:=\frac{1}{2 \pi \rho} \int_{0}^{2 \pi \rho} \mathrm{d} \tau \mathrm{e}^{\tilde{F}(\tau)} b(\tau)\left[\dot{u}_{0}(\tau) h\left(u_{0}(\tau)\right)+\frac{2}{\rho \Omega_{0}} k\left(u_{0}(\tau)\right)\right]
$$

and

$$
\begin{aligned}
& B_{i 1 \nu}:=\frac{1}{2 \pi p} \int_{0}^{2 \pi p} \mathrm{~d} \tau \frac{K_{i}(\tau)}{\rho^{2} \Omega_{0}^{2}} \sin \nu \tau, \quad i=1,2, B_{31 \nu}:=\nu \rho \Omega_{0} B_{22 \nu}, \\
& B_{i 2 \nu}:=\frac{1}{2 \pi p} \int_{0}^{2 \pi p} \mathrm{~d} \tau \frac{K_{i}(\tau)}{\rho^{2} \Omega_{0}^{2}} \cos \nu \tau, \quad i=1,2, \quad B_{32 \nu}:=-\nu \rho \Omega_{0} B_{21 \nu},
\end{aligned}
$$


with

$$
K_{1}(\tau)=\mathrm{e}^{\tilde{F}(\tau)} b(\tau) \rho \Omega_{0} v_{0}(\tau)\left(3 u_{0}^{2}(\tau)-1\right), \quad K_{2}(\tau)=\mathrm{e}^{\tilde{F}(\tau)} b(\tau) u_{0}(\tau)\left(u_{0}^{2}(\tau)-1\right) .
$$

By setting $D_{1 \nu}=-\left(B_{11 \nu}+B_{21 \nu}+B_{31 \nu}\right)$ and $D_{2 \nu}=-\left(B_{12 \nu}+B_{22 \nu}+B_{32 \nu}\right)$, (2.23) then becomes

$$
\varepsilon_{1}\left(\tau_{0}\right)=\frac{1}{A} \sum_{\nu=1}^{\infty} \hat{f}_{\nu}\left(D_{1 \nu} \cos \nu \tau_{0}+D_{2 \nu} \sin \nu \tau_{0}\right):=\mathfrak{D}_{1}\left(\tau_{0}\right) .
$$

By construction $\varepsilon_{1}$ has zero mean, so that either it is a non-constant function or it identically vanishes. For purposes of comparison with [4, in the following we shall shorten $D_{11}=D_{1}$ and $D_{21}=D_{2}$, and also $B_{i j 1}=B_{i j}$, which are the only relevant constants when $f$ contains only the first harmonics $\nu=1$ in (2.4).

It is shown in Appendix B of [4] that $A=-r_{1} \rho \Omega_{0}$; hence, from (2.17),

$$
A=\frac{U_{0}\left(\alpha-\beta+\beta U_{0}^{2}\right)}{\rho \Omega_{0}},
$$

which provides an obvious means to check the numerics — by calculating $A$ from (2.24) and comparing with (2.28).

In 4 it is also shown how to go to higher orders; to any order $k \geq 1$ one finds the compatibility condition $\left\langle\mathrm{e}^{\tilde{F}} b \Psi_{k}\right\rangle=0$, where the function $\Psi_{k}(\tau)$ is given by (2.21).

The compatibility condition leads to all orders to equations like (2.27), which now read

$$
\varepsilon_{k}\left(\tau_{0}\right)=\mathfrak{D}_{k}\left(\tau_{0}\right), \quad k \geq 1,
$$

for suitable functions $\mathfrak{D}_{k}$ - strictly speaking in [4 only the case $f(t)=\sin t$ is explicitly discussed, but one can easily work out the general case of $f$ an arbitrary analytic function by following the same strategy. Note that, with respect to [4, here we have included the factor $1 / A$ in the definition of $\mathfrak{D}_{k}\left(\tau_{0}\right)$.

The width of the plateau corresponding to a given $\rho$ (i.e. to a given resonance $p: q$ such that $\rho=p / q)$ can then be expressed as follows. First one defines

$$
\mathfrak{D}\left(\tau_{0}, \mu\right)=\sum_{k=1}^{\infty} \varepsilon^{k} \mathfrak{D}_{k}\left(\tau_{0}\right), \quad \varepsilon_{\max }(\rho):=\max _{0 \leq \tau_{0} \leq 2 \pi} \mathfrak{D}\left(\tau_{0}, \mu\right), \quad \varepsilon_{\min }(\rho):=\min _{0 \leq \tau_{0} \leq 2 \pi} \mathfrak{D}\left(\tau_{0}, \mu\right) .
$$

Then by setting

$$
\omega_{\min }(\rho):=\frac{\rho \Omega_{0}}{1+\rho \Omega_{0} \varepsilon_{\max }(\rho)}, \quad \omega_{\max }(\rho):=\frac{\rho \Omega_{0}}{1+\rho \Omega_{0} \varepsilon_{\min }(\rho)},
$$

the plateau corresponding to $\rho$ is given by

$$
\Delta \omega(\rho):=\omega_{\max }(\rho)-\omega_{\min }(\rho)=\frac{\rho^{2} \Omega_{0}^{2}\left(\varepsilon_{\max }(\rho)-\varepsilon_{\min }(\rho)\right)}{\left(1+\rho \Omega_{0} \varepsilon_{\min }(\rho)\right)\left(1+\rho \Omega_{0} \varepsilon_{\max }(\rho)\right)} .
$$

In other words, for $\omega \in\left[\omega_{\min }(\rho), \omega_{\max }(\rho)\right]$, one has locking $\omega=\rho \Omega$, if $\Omega$ denotes the frequency of the output signal. For each such value of $\omega$ the initial phase $\tau_{0}$ gets fixed to a value $\tau_{0}^{*}$ such that $1 / \omega=1 / \rho \Omega_{0}+\varepsilon\left(\mu, \tau_{0}^{*}\right)$, according to (2.9).

When the function $\varepsilon_{1}\left(\tau_{0}\right)$ in (2.27) does not vanish, then, if one further assumes that the second derivative of $\mathfrak{D}_{1}$ is non-zero at the stationary points (where the maximum and minimum are attained), the first order approximation is adequate. In other words, in such a case one can approximate

$$
\varepsilon_{\max }(\rho)=\mu \max _{0 \leq \tau_{0} \leq 2 \pi} \mathfrak{D}_{1}\left(\tau_{0}\right)+O\left(\mu^{2}\right), \quad \varepsilon_{\min }(\rho)=\mu \min _{0 \leq \tau_{0} \leq 2 \pi} \mathfrak{D}_{1}\left(\tau_{0}\right)+O\left(\mu^{2}\right),
$$


and hence

$$
\begin{aligned}
& \omega_{\min }(\rho)=\rho \Omega_{0}\left(1-\rho \Omega_{0} \mu \max _{0 \leq \tau_{0} \leq 2 \pi} \mathfrak{D}_{1}\left(\tau_{0}\right)\right)+O\left(\mu^{2}\right), \\
& \omega_{\max }(\rho)=\rho \Omega_{0}\left(1-\rho \Omega_{0} \mu \min _{0 \leq \tau_{0} \leq 2 \pi} \mathfrak{D}_{1}\left(\tau_{0}\right)\right)+O\left(\mu^{2}\right),
\end{aligned}
$$

which gives a plateau of width

$$
\Delta \omega(\rho)=\mu \Delta_{1} \omega(\rho)+O\left(\mu^{2}\right), \quad \Delta_{1} \omega(\rho):=\rho^{2} \Omega_{0}^{2}\left(\max _{0 \leq \tau_{0} \leq 2 \pi} \mathfrak{D}_{1}\left(\tau_{0}\right)-\min _{0 \leq \tau_{0} \leq 2 \pi} \mathfrak{D}_{1}\left(\tau_{0}\right)\right)
$$

centred 'around' the value $\omega_{\mathrm{c}}(\rho)=\rho \Omega_{0}$. Since the function $\varepsilon_{1}\left(\tau_{0}\right)$ has zero mean, this means that the corresponding Arnol'd tongue in the $(\omega, \mu)$ plane emanates from the point $\omega_{\mathrm{c}}(\rho)$ of the $\omega$-axis as a cone with axis along the vertical and angle $\theta(\rho)=\theta_{1}(\rho)+\theta_{2}(\rho)$ such that

$$
\tan \theta_{1}(\rho)=-\rho^{2} \Omega_{0}^{2} \min _{0 \leq \tau_{0} \leq 2 \pi} \mathfrak{D}_{1}\left(\tau_{0}\right), \quad \tan \theta_{2}(\rho)=\rho^{2} \Omega_{0}^{2} \max _{0 \leq \tau_{0} \leq 2 \pi} \mathfrak{D}_{1}\left(\tau_{0}\right) .
$$

If $f$ contains only one harmonic, say $\hat{f}_{\nu}=0$ for $|\nu|>1$ in (2.4), then $\theta_{1}(\rho)=\theta_{2}(\rho)$, and $\max \mathfrak{D}_{1}\left(\tau_{0}\right)$ $=A^{-1} \sqrt{D_{1}^{2}+D_{2}^{2}}$. Note that in such a case the second derivative of $\mathfrak{D}_{1}$ equals $\pm \hat{f}_{1} / A$ when the first derivative vanishes.

\section{$3 \quad$ Arnol'd Tongues: analytical results}

\subsection{First order contributions}

Let us consider the expression in (2.32) for the leading contribution to the width of the plateau when the first order contribution does not vanish. Then we neglect the high order terms and approximate

$$
\Delta \omega(\rho) \approx \mu \rho^{2} \Omega_{0}^{2} Q(\rho), \quad \text { where } \quad Q(\rho)=\max _{0 \leq \tau_{0} \leq 2 \pi} \mathfrak{D}_{1}\left(\tau_{0}\right)-\min _{0 \leq \tau_{0} \leq 2 \pi} \mathfrak{D}_{1}\left(\tau_{0}\right),
$$

Note that, to obtain $\mathfrak{D}_{1}\left(\tau_{0}\right)$ from (2.27), one must keep only the summands such that $\hat{f}_{\nu} \neq 0$.

By writing $B_{i j \nu}$ according to (2.25), one uses that the Fourier expansions of the functions $K_{i}$ contain only even harmonics (cf. Section 6 in [4]), i.e.

$$
K_{i}(\tau)=\sum_{\substack{\nu^{\prime} \in \mathbb{Z} \\ \nu^{\prime} \text { even }}} \mathrm{e}^{\mathrm{i} \nu^{\prime} \tau / \rho} \hat{K}_{i \nu^{\prime}}=\sum_{\nu^{\prime} \in \mathbb{Z}} \mathrm{e}^{\mathrm{i} 2 \nu^{\prime} \tau / \rho} \hat{K}_{i\left(2 \nu^{\prime}\right)} .
$$

Furthermore, as (2.26) shows, the functions $K_{i}$ are analytic and hence the corresponding Fourier coefficients $\hat{K}_{i \nu^{\prime}}$ decay exponentially, i.e. for $i=1,2$ and for all $\nu^{\prime} \in \mathbb{Z}$ one has $\left|\hat{K}_{i \nu^{\prime}}\right| \leq \Gamma \mathrm{e}^{-\xi_{1}\left|\nu^{\prime}\right|}$ for suitable positive constants $\Gamma$ and $\xi_{1}$.

Hence by expanding $K_{i}$ according to (3.2) and writing

$$
\sin \nu \tau=\sum_{\sigma= \pm 1} \frac{\sigma}{2 \mathrm{i}} \mathrm{e}^{\mathrm{i} \sigma \nu \tau}, \quad \cos \nu \tau=\sum_{\sigma= \pm 1} \frac{1}{2} \mathrm{e}^{\mathrm{i} \sigma \nu \tau}
$$

one realises that one can have $B_{i j \nu} \neq 0$ only if there exist $\nu^{\prime} \in \mathbb{Z}$ such that $\hat{K}_{i \nu^{\prime}} \neq 0$ and $\nu^{\prime} q+\sigma \nu p=0$. If we assume that the first condition is satisfied for all even $\nu^{\prime} \in \mathbb{Z}$ (numerical analysis ensures that such an assumption is reasonable - see figure 1), then the key condition is that there exist $\nu^{\prime} \in \mathbb{Z}$ such that

$$
2\left|\nu^{\prime}\right| q=|\nu| p
$$


with $p, q$ relatively prime integers. When this happens one has

$$
B_{i j \nu}=\frac{1}{\rho^{2} \Omega_{0}^{2}} \sum_{\substack{\nu^{\prime} \in \mathbb{Z}, \sigma= \pm 1 \\ 2 \nu^{\prime}+\sigma \nu \rho=0}} \hat{K}_{i\left(2 \nu^{\prime}\right)} \hat{R}_{j \sigma}, \quad i, j=1,2, \quad \text { where } \quad \hat{R}_{1 \sigma}=\frac{\sigma}{2 \mathrm{i}}, \quad \hat{R}_{2 \sigma}=\frac{1}{2},
$$

and $B_{31 \nu}=\nu \rho \Omega_{0} B_{22 \nu}, B_{32 \nu}=-\nu \rho \Omega_{0} B_{21 \nu}$. If the function $f$ contains only the first harmonics (so that $\hat{f}_{\nu} \neq 0$ only for $|\nu|=1$ ) then in (3.4) one has to consider only the case $|\nu|=1$. Thus, as discussed already in 4 , one obtains $q=1$ and $p=2\left|\nu^{\prime}\right|$, i.e. $p$ must be even. This means that one finds plateaux of width $O(\mu)$ only for resonances $p: q$ with $q=1$ and $p \in 2 \mathbb{N}$.

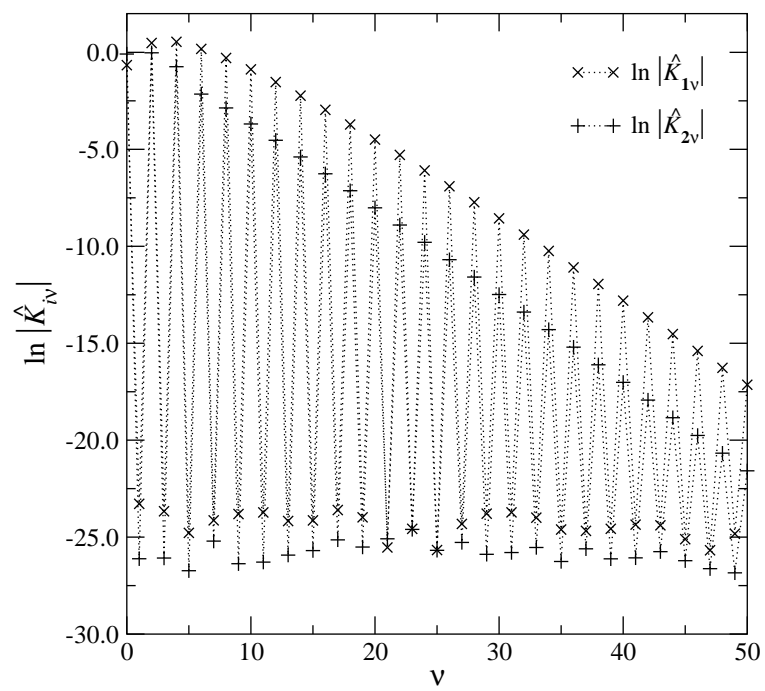

Figure 1: Fourier coefficients of the functions $K_{1}(\tau), \times$, and $K_{2}(\tau),+$, for $\alpha=5, \beta=4$. The odd coefficients turn out to be zero (within the numerical error of $\sim 10^{-11}$ ), according to (3.2), while all the even coefficients are non-zero and decay exponentially. The dotted lines are only to guide the eye.

On the contrary, if the function $f$ contains all the harmonics, the condition (3.4) has to be considered for all $\nu \in \mathbb{Z}$, and one finds easily non-vanishing contributions to (3.5), e.g. by taking $\left|\nu^{\prime}\right|=p$ and $|\nu|=2 q$. Thus, for any resonance $p: q$ one has a plateau which to first order is given by (3.1). From (2.23) one obtains

$$
\mathfrak{D}_{1}\left(\tau_{0}\right)=-\frac{1}{A \rho^{2} \Omega_{0}^{2}} \sum_{\substack{\nu \geq 1 \\ \nu \rho \text { even }}} \hat{f}_{\nu}\left(\bar{K}_{1(\nu \rho)} \cos \nu \tau_{0}+\bar{K}_{2(\nu \rho)} \sin \nu \tau_{0}\right),
$$

where we have defined

$$
\begin{aligned}
& \bar{K}_{1 \nu}:=\sum_{\sigma= \pm 1}\left[\hat{R}_{1 \sigma}\left(\hat{K}_{1(-\sigma \nu)}+\hat{K}_{2(-\sigma \nu)}\right)+\nu \rho \Omega_{0} \hat{R}_{2 \sigma} \hat{K}_{2(-\sigma \nu)}\right], \\
& \bar{K}_{2 \nu}:=\sum_{\sigma= \pm 1}\left[\hat{R}_{2 \sigma}\left(\hat{K}_{1(-\sigma \nu)}+\hat{K}_{2(-\sigma \nu)}\right)-\nu \rho \Omega_{0} \hat{R}_{1 \sigma} \hat{K}_{2(-\sigma \nu)}\right],
\end{aligned}
$$

Let us define $\nu_{0}=\min \{\nu \geq 1: \nu \rho$ even $\}$ and $\nu_{1}=\min \left\{\nu>\nu_{0}: \nu \rho\right.$ even $\}$, and set

$$
\bar{K}_{\nu}(\rho):=\sqrt{\left|\bar{K}_{1 \nu}\right|^{2}+\left|\bar{K}_{2 \nu}\right|^{2}}, \quad Q_{0}(\rho)=\frac{2}{|A| \rho^{2} \Omega_{0}^{2}}\left|\hat{f}_{\nu_{0}}\right|\left|\bar{K}_{\nu_{0} \rho}(\rho)\right| .
$$

Then one obtains

$$
Q(\rho)=Q_{0}(\rho)+O\left(\frac{\left|\hat{f}_{\nu_{1}}\right|\left|\bar{K}_{\nu_{1} \rho}(\rho)\right|}{\left|\hat{f}_{\nu_{0}}\right|\left|\bar{K}_{\nu_{0} \rho}(\rho)\right|}\right)
$$


which inserted into (3.1) gives

$$
\Delta \omega(\rho) \approx \frac{2 \mu \rho \Omega_{0}}{\left|\bar{r}_{1}\right|}\left|\hat{f}_{\nu_{0}}\right|\left|\bar{K}_{\nu_{0} \rho}(\rho)\right|,
$$

where we have used that $A=-\bar{r}_{1} / \rho \Omega_{0}$, with the constant $\bar{r}_{1}$ independent of $\rho \Omega_{0}$.

If one keeps the whole sum in (3.6) one finds, always in the first order approximation,

$$
|\Delta \omega(\rho)| \leq \frac{2 \mu \rho \Omega_{0}}{\left|\bar{r}_{1}\right|} \max _{i=1,2} \sum_{\nu=1}^{\infty}\left|\hat{f}_{\nu}\right|\left|\bar{K}_{i(\nu \rho)}(\rho)\right| \leq \frac{2 \mu \rho \Omega_{0}}{\left|\bar{r}_{1}\right|} \max _{i=1,2} \sum_{\substack{\nu \in \mathbb{Z} \\ \nu \rho \text { even }}}\left(1+\nu \rho \Omega_{0}\right)\left|\hat{f}_{\nu}\right|\left|\hat{K}_{i(\nu \rho)}(\rho)\right| .
$$

Since $p$ and $q$ are relatively prime the condition $\nu \rho \in 2 \mathbb{Z}$ can be satisfied only if $|\nu| \geq q$ and $|\nu \rho| \geq p$. Therefore for fixed $\rho=p / q$ one has

$$
|\Delta \omega(\rho)| \leq \mu C p^{2} q^{-1} \mathrm{e}^{-\xi_{1} p} \mathrm{e}^{-\xi q},
$$

where $C$ is a constant independent of $\rho$. This shows that all the Arnol'd tongues have width proportional to $\mu$, but the constant of proportionality decays exponentially with $p$ and $q$. Therefore, for fixed $\mu$, the union of all the Arnol'd tongues is $O(\mu)$, and hence tends to zero when $\mu \rightarrow 0$, as expected from [11.

For instance, if $f(t)=\sin t+\eta \sin 2 t$ in (2.4), one has $\Delta(2 n)=c(2 n) \mu+O\left(\mu^{2}\right)$ and $\Delta(2 n-1)=$ $c(2 n-1) \eta \mu+O\left(\mu^{2}\right)$, for suitable constants $c(n)$ independent of $\mu$ and $\eta$. Therefore, for all integer resonances the plateaux are of the same order of magnitude - provided, of course, $\eta$ is large compared with $\mu$.

\subsection{Second order contributions}

When the first order dominates, the second order gives a correction which can be computed explicitly. When the first order vanishes, the second order becomes the leading order (if it does not vanish too).

To compute the second order one needs the function $\mathfrak{D}_{2}$ appearing in (2.29) for $k=2$. The analysis in [4] and (2.29) show that

$$
\left\langle\mathrm{e}^{\tilde{F}} b \Psi_{2}\right\rangle=A \varepsilon_{2}\left(\tau_{0}\right)+\left\langle\mathrm{e}^{\tilde{F}} b \Xi_{2}\left(\cdot ; \tau_{0}\right)\right\rangle \quad \Longrightarrow \quad \mathfrak{D}_{2}\left(\tau_{0}\right)=-\frac{1}{A}\left\langle\mathrm{e}^{\tilde{F}} b \Xi_{2}\left(\cdot ; \tau_{0}\right)\right\rangle,
$$

where, by (2.11) and (2.21) with $k=2$, one can write

$$
\begin{aligned}
\Xi_{2}\left(\tau ; \tau_{0}\right) & =\widetilde{\Xi}_{2}\left(\tau ; \tau_{0}\right)+\bar{\Xi}_{2}\left(\tau ; \tau_{0}\right), \\
\widetilde{\Xi}_{2}\left(\tau ; \tau_{0}\right) & =-\varepsilon_{1}^{2}\left(\tau_{0}\right)\left[(\alpha-\beta) u_{0}(\tau)+\beta u_{0}^{3}(\tau)\right]-\varepsilon_{1}\left(\tau_{0}\right) v_{0}(\tau)\left(3 u_{0}^{2}(\tau)-1\right) f\left(\tau+\tau_{0}\right) \\
& -\varepsilon_{1}\left(\tau_{0}\right)\left(u_{0}^{3}(\tau)-u_{0}(\tau)\right)\left(\frac{2}{\rho \Omega_{0}} f\left(\tau+\tau_{0}\right)+f^{\prime}\left(\tau+\tau_{0}\right)\right), \\
\Xi_{2}\left(\tau ; \tau_{0}\right) & =-u_{1}(\tau) \frac{\partial H_{1}}{\partial u_{0}}\left(u_{0}(\tau), v_{0}(\tau), \tau+\tau_{0}\right)-\dot{u}_{1}(\tau) \frac{\partial H_{1}}{\partial \dot{u}_{0}}\left(u_{0}(\tau), v_{0}(\tau), \tau+\tau_{0}\right) \\
& +\frac{1}{2} u_{1}^{2}(\tau) \frac{\partial^{2} G}{\partial u^{2}}\left(u_{0}(\tau), v_{0}(\tau)\right)+u_{1}(\tau) \dot{u}_{1}(\tau) \frac{\partial^{2} G}{\partial u \partial v}\left(u_{0}(\tau), v_{0}(\tau)\right),
\end{aligned}
$$

with $H_{1}(u, v, \tau)$ and $G(u, v)$ given in (2.11b) and (2.12), respectively (we have explicitly used that $G(u, v)$ is linear in $v)$.

Thus, to compute (3.13) one first needs the first order solution $\left(u_{1}, v_{1}\right)$, with $v_{1}(\tau)=\dot{u}_{1}(\tau)$. For $k=1$ equation (2.20) gives

$$
u_{1}(\tau)=c a(\tau)\left(\mathcal{Q}_{2}(\tau)-\mathcal{Q}_{2}(0)-\mathcal{Q}_{1}(0)\right)-c b(\tau) \mathcal{Q}_{1}(\tau),
$$


where the functions $\mathcal{Q}_{1}$ and $\mathcal{Q}_{2}$ can be read from equations (5.3) and (5.4) of [4], which we rewrite here for convenience,

$$
\int_{0}^{\tau} \mathrm{d} \tau^{\prime} \mathrm{e}^{F\left(\tau^{\prime}\right)} a\left(\tau^{\prime}\right) \Psi_{1}\left(\tau^{\prime}\right)=\mathrm{e}^{f_{0} \tau} \mathcal{Q}_{1}(\tau)-\mathcal{Q}_{1}(0), \quad \int_{0}^{\tau} \mathrm{d} \tau^{\prime} \mathrm{e}^{\tilde{F}\left(\tau^{\prime}\right)} b\left(\tau^{\prime}\right) \Psi_{1}\left(\tau^{\prime}\right)=\mathcal{Q}_{2}(\tau)-\mathcal{Q}_{2}(0),
$$

and we are using that $\mathcal{Q}_{0}:=\left\langle\mathrm{e}^{\tilde{F}} b \Psi_{1}\right\rangle=0$ and $\bar{u}_{1}=-c \mathcal{Q}_{1}(0)$. Note that the functions $u_{1}$ and $v_{1}$ depend also on $\tau_{0}$; more precisely, by construction one has

$$
u_{1}(\tau)=\sum_{\substack{\nu \in \mathbb{Z} \\ \nu \text { odd }}} \sum_{\nu_{1} \in \mathbb{Z}} \mathrm{e}^{\mathrm{i} \nu \tau / \rho} \mathrm{e}^{\mathrm{i} \nu_{1}\left(\tau+\tau_{0}\right)} \hat{U}_{1 \nu \nu_{1}}, \quad \hat{U}_{1 \nu \nu_{1}} \propto \hat{f}_{\nu_{1}}
$$

as easily follows by reasoning as in the proof of lemma 8.2 in [4, the only difference being that $f$ can contain all harmonics. to

In particular, when $\varepsilon_{1}$ vanishes identically, then $\widetilde{\Xi}_{2}$ also is identically zero and (3.13) reduces

$$
\begin{aligned}
\mathfrak{D}_{2}\left(\tau_{0}\right) & =\frac{1}{A} \frac{1}{2 \pi p} \int_{0}^{2 \pi p} \mathrm{~d} \tau \mathrm{e}^{\tilde{F}(\tau)} b(\tau) \frac{1}{\rho \Omega_{0}}\left\{\left[\left(6 u_{0}(\tau) v_{0}(\tau)+\frac{1}{\rho \Omega_{0}}\left(3 u_{0}^{2}(\tau)-1\right)\right) f\left(\tau+\tau_{0}\right)\right.\right. \\
& \left.+\left(3 u_{0}^{2}(\tau)-1\right) f^{\prime}\left(\tau+\tau_{0}\right)\right] u_{1}(\tau)+\left(3 u_{0}^{2}(\tau)-1\right) f\left(\tau+\tau_{0}\right) v_{1}(\tau) \\
& \left.+\frac{1}{2}\left(v_{0}(\tau) h^{\prime \prime}\left(u_{0}(\tau)\right)+\frac{k^{\prime \prime}\left(u_{0}(\tau)\right)}{\rho \Omega_{0}}\right) u_{1}^{2}(\tau)+h^{\prime}\left(u_{0}(\tau)\right) u_{1}(\tau) v_{1}(\tau)\right\},
\end{aligned}
$$

where $h^{\prime}(u)=6 \beta u, h^{\prime \prime}(u)=6 \beta$, and $k^{\prime \prime}(u)=6 \beta u$ (here, as well as for $f$, the prime denotes derivative with respect to the argument).

By using the expansion (3.17) for $u_{1}$, one finds that the function

$$
\mathfrak{D}_{2}\left(\tau_{0}\right)=\sum_{\nu \in \mathbb{Z}} \mathrm{e}^{\mathrm{i} \nu \tau_{0}} \mathfrak{D}_{2, \nu}=\mathfrak{D}_{2,0}+\sum_{\substack{\nu \in \mathbb{Z} \\ \nu \neq 0}} \mathrm{e}^{\mathrm{i} \nu \tau_{0}} \mathfrak{D}_{2, \nu}
$$

can be written in the form

$$
\mathfrak{D}_{2}\left(\tau_{0}\right)=\frac{1}{2 \pi p} \sum_{\substack{\nu^{\prime} \in \mathbb{Z} \nu_{1}, \nu_{2} \in \mathbb{Z} \\ \nu^{\prime} \text { even }}} \int_{0}^{2 \pi p} \mathrm{~d} \tau \mathrm{e}^{\mathrm{i} \nu^{\prime} \tau / \rho} \mathrm{e}^{\mathrm{i}\left(\nu_{1}+\nu_{2}\right)\left(\tau+\tau_{0}\right)} \hat{\mathfrak{K}}_{\nu^{\prime} \nu_{1} \nu_{2}}, \quad \hat{\mathfrak{K}}_{\nu^{\prime} \nu_{1} \nu_{2}} \propto \mathrm{e}^{-\xi_{1}\left|\nu^{\prime}\right|} \hat{f}_{\nu_{1}} \hat{f}_{\nu_{2}},
$$

for suitable coefficients $\hat{\mathfrak{K}}_{\nu^{\prime} \nu_{1} \nu_{2}}$. Then one sees that only the coefficients $\hat{\mathfrak{K}}_{\left(2 \nu^{\prime}\right) \nu_{1} \nu_{2}}$ with

$$
2\left|\nu^{\prime}\right| q=\left|\nu_{1}+\nu_{2}\right| p, \quad \hat{f}_{\nu_{1}} \hat{f}_{\nu_{2}} \neq 0
$$

contribute to (3.20). The term with $\nu_{1}+\nu_{2}=\nu^{\prime}=0$ gives the mean $\mathfrak{D}_{2,0}$ of $\mathfrak{D}_{2}$, and requires no condition on $\rho$. This explains why the boundaries of the locking region are either $O(\mu)$ - when the first order dominates - or $O\left(\mu^{2}\right)$ - in all the other cases. However, the width of the plateau arises from the variations of $\mathfrak{D}_{2}$, hence it is related to the terms in (3.20) with $\nu \neq 0$ such that (3.21) is satisfied. If there are any of such terms, then the function $\mathfrak{D}_{2}$ is not identically constant, and therefore, in such a case, one has

$$
\Delta \omega(\rho)=\mu^{2} \Delta_{2} \omega(\rho)+O\left(\mu^{3}\right), \quad \Delta_{2} \omega(\rho):=\rho^{2} \Omega_{0}^{2}\left(\max _{0 \leq \tau_{0} \leq 2 \pi} \mathfrak{D}_{2}\left(\tau_{0}\right)-\min _{0 \leq \tau_{0} \leq 2 \pi} \mathfrak{D}_{2}\left(\tau_{0}\right)\right)
$$

which replaces (2.35) when the first order vanishes. For instance if $f$ contains only the first harmonics then (3.21) is satisfied for $q=1, p \in \mathbb{N}$ and $\nu_{1}=\nu_{2}= \pm 1$, which shows that the plateaux corresponding to odd $\rho$ are of order $\mu^{2}$ - see [4] for further details. 


\subsection{Higher order contributions}

If one wants to determine the higher order contributions, the analysis above can be easily extended, even if it becomes much more complicated from the computational point of view. In general, if one writes

$$
\Delta \omega(\rho)=\sum_{k=1}^{\infty} \mu^{k} \Delta_{k} \omega(\rho),
$$

one finds

$$
\Delta_{k} \omega(\rho)=\sum_{\substack{\nu \in \mathbb{Z} \\\left|\nu_{1}+\ldots+\nu_{k}\right| p=2 \nu q}} \sum_{\substack{\nu_{1}, \ldots, \nu_{k} \in \mathbb{Z} \\ \mid \nu_{k}}} \Delta_{k} \omega\left(\rho ; \nu_{1}, \ldots, \nu_{k}\right), \quad \Delta_{k} \omega\left(\rho ; \nu_{1}, \ldots, \nu_{k}\right) \propto \mathrm{e}^{-2 \xi_{1}|\nu| / \rho} \prod_{i=1}^{k} \hat{f}_{\nu_{i}}
$$

so that, in order to single out the leading contribution to the width of the plateau, one has to compare the size of the perturbation parameter $\mu$ with the amplitudes of the harmonics $\hat{f}_{\nu}$ of the drive.

Note that to all orders $k$ the coefficients $\Delta_{k} \omega(\rho)$ decay exponentially in both $p$ and $q$. Thus, every time the first order does not vanish it dominates, provided $\mu$ is small enough. If on the contrary $\Delta_{k^{\prime}} \omega(\rho)=0$ for all $1 \leq k^{\prime}<k$ and $\Delta_{k} \omega(\rho) \neq 0$ then one has $\Delta \omega(\rho)=O\left(\mu^{k}\right)$ for $\mu$ small enough.

\section{Numerical computations}

\subsection{Numerical solution of the ODE}

Since in general no closed-form solution to (2.7) with $\mu=0$ exists for $\beta \neq 0$, it is clear that this equation must be solved numerically. Furthermore, in order to approximate $u_{0}(\tau)$ and $\dot{u}_{0}(\tau)$, the ODE must be solved for a sufficiently long time that the transient has, for practical purposes, decayed to zero. An effective initial procedure was found to be (i) solve the ODE from $\tau=-\tau_{1}$ to $\tau=0$, where $\tau_{1}$ is large, using any standard method, for example, the Runge-Kutta fourth order method; (ii) solve for a further small time $\tau_{2}$ which is such that $\dot{u}_{0}\left(\tau_{2}\right)=0$ and $u_{0}\left(\tau_{2}\right)>0$, again using the Runge-Kutta method, and additionally using bisection to find $\tau_{2}$ such that the first condition is met; (iii) solve from $\tau=\tau_{2}$ to $\tau_{3}$, where $\tau_{3}$ is the smallest value of $\tau$ which is greater than $\tau_{2}$ and for which, again, $\dot{u}_{0}\left(\tau_{3}\right)=0$ and $u_{0}\left(\tau_{3}\right)>0$. Then an estimate of the period of $u_{0}(\tau), T_{0}$, is $\tau_{3}-\tau_{2}$ and an estimate of $U_{0}$ is $u_{0}\left(\tau_{2}\right) \approx u_{0}\left(\tau_{3}\right)$.

In practice, these estimates can then be somewhat improved by solving the ODE assuming that a power series for $u_{0}(\tau)$ exists, and computing this series around $\tau=0$, using the initial conditions $u_{0}(0)=U_{0}$, as estimated above, and $\dot{u}_{0}(0)=0$. We can shift the origin of time from $\tau_{3}$ to zero because the ODE is autonomous. Typically, several power series need to be computed to cover the range $\tau=0$ to $T_{0}$, but the method has at least two advantages over Runge-Kutta. The first is that the error can be estimated by implementing a test on the coefficients of the power series, as set out in [5]; the second is that the Newton-Raphson method can be used directly on the power series for the solution around $T_{0}$ to find the value of $\tau$ for which $\dot{u}_{0}(\tau)=0$, and hence to estimate $T_{0}$. The series used in practical computations had degree 30.

Once accurate values of $U_{0}$ and $T_{0}$ have been computed, it is a simple matter to calculate a table of values of $u_{0}(\tau)$ and $\dot{u}_{0}(\tau)$ at $\tau=i h, i=0 \ldots M-1$ for some $M \in \mathbb{N}$ and for $h=T_{0} / M>0$ a given time-step.

\subsection{Interpolation}

A discussion of a suitable interpolation method is now in order. 
In what follows, we will need to integrate functions of $u_{0}(\tau), \dot{u}_{0}(\tau)$ and to do this we use an interpolation scheme from which such integrals can be computed directly.

We start by discussing a scheme for interpolating from the values of $u_{0}(\tau), \dot{u}_{0}(\tau)$ at discrete times $i h, i=0 \ldots M-1$, produced by the numerical ODE solver.

Since $u_{0}(\tau)$ is periodic, the interpolation scheme should reflect this — standard methods based on the Lagrange formula or Chebyshev polynomial interpolation are therefore not suitable. Instead, interpolation based on the function

$$
I_{K}(\tau)=\frac{\sin K \pi \tau}{K \sin \pi \tau}
$$

where $K$ is an odd, positive integer, is used. This function is equivalent to a truncated Fourier series (see Appendix A) and has the properties that (i) $I_{K}(\tau+1)=I_{K}(\tau)$, so it is periodic (if $K$ is even, the period is not 1 but 2); and (ii)

$$
\lim _{\substack{\tau \rightarrow n \\ n \in \mathbb{Z}}} I_{K}(\tau)=1 \quad \text { and } \quad I_{K}\left(\frac{m}{K}\right)=0, \quad m \in \mathbb{Z}, m \text { not a multiple of } K .
$$

Now let $x(\tau)=x\left(\tau+T_{0}\right)$ be a periodic function of $\tau$ with period $T_{0}$ and set $x_{j}=x\left(j T_{0} / K\right)$ for $j=0 \ldots K-1$. Then, defining

$$
\widehat{x}(\tau):=\sum_{j=0}^{K-1} x_{j} I_{K}\left(\tau / T_{0}-j / K\right),
$$

we have, in the light of (i) and (ii) above, that $\widehat{x}\left(k T_{0} / K\right)=x_{k}=x\left(k T_{0} / K\right)$ for $k \in \mathbb{Z}$. Hence, $\widehat{x}(\tau)$ can be used to interpolate $x(\tau)$ given the values of $x(\tau)$ on a uniformly-spaced discrete set of values of $\tau$. In Appendix $\mathrm{A}$ we show that the error in the interpolation scheme described is $O\left(\mathrm{e}^{-C_{2} K}\right)$, for some positive constant $C_{2}$.

In practice, for $\tau$ close to an integer, $I_{K}(\tau)$ is best computed from a series expansion. Letting $\delta=\tau-[\tau]$, with $[\tau]$ being the nearest integer to $\tau$, we then use

$$
I_{K}(\delta)=1-\frac{1}{6}\left(K^{2}-1\right)\left[(\pi \delta)^{2}-\frac{1}{60}\left(3 K^{2}-7\right)(\pi \delta)^{4}+\frac{1}{2520}\left(3 K^{4}-18 K^{2}+31\right)(\pi \delta)^{6}\right]+O\left(\delta^{8}\right)
$$

whenever $|\delta|<\varepsilon_{I}$. Since the computations are carried out to approximately 16 significant figures, we allow a margin of safety by choosing $\varepsilon_{I}=10^{-4}$.

The use of $I_{K}(\tau)$ for interpolation has other advantages, amongst them that $\widehat{x}(\tau)$ can be integrated in closed form, and so, by implication, the integral of $x(\tau)$ for all $\tau$ can be approximated. By defining

$$
J_{K}^{\prime}(T)=\int_{0}^{T} \mathrm{~d} \tau \frac{\sin K \pi \tau}{\sin \pi \tau}
$$

it is easy to show that

$$
J_{K+2}^{\prime}(T)=J_{K}^{\prime}(T)+2 \int_{0}^{T} \mathrm{~d} \tau \cos (K+1) \pi \tau=J_{K}^{\prime}(T)+\frac{2}{(K+1) \pi} \sin (K+1) \pi T .
$$

Now, since $K>0$ is odd and $J_{1}^{\prime}(T)=T$, we have

$$
J_{K}^{\prime}(T)=T+\frac{1}{\pi} \sum_{i=1}^{(K-1) / 2} \frac{1}{i} \sin 2 i \pi T .
$$

Defining now $J_{K}(T):=\int_{0}^{T} \mathrm{~d} \tau I_{K}(\tau)=J_{K}^{\prime}(T) / K$, we have

$$
J_{K}(T)=\frac{T}{K}+\frac{1}{K \pi} \sum_{i=1}^{(K-1) / 2} \frac{1}{i} \sin 2 i \pi T .
$$


Next define $\widehat{X}(T)=\int_{0}^{T} \mathrm{~d} \tau \widehat{x}(\tau)$. Integrating (4.2) term-by-term, we obtain

$$
\widehat{X}(T)=T_{0} \sum_{i=0}^{K-1} x_{i}\left\{J_{K}\left(\frac{T}{T_{0}}-\frac{i}{K}\right)+J_{K}\left(\frac{i}{K}\right)\right\},
$$

where we have used the fact that $J_{K}(\tau)$ is an odd function of $\tau$. In what follows, we therefore use $\widehat{X}(T)$ to approximate $\int_{0}^{T} \mathrm{~d} \tau x(\tau)$.

In a similar manner, defining $E_{K}(\zeta, T):=\int_{0}^{T} \mathrm{~d} \tau \mathrm{e}^{-\zeta \tau} I_{K}(\tau)$, for constant $\zeta$, it can be shown that

$$
E_{K}(\zeta, T)=\frac{1-\mathrm{e}^{-\zeta T}}{\zeta K}+\frac{2}{K} \sum_{i=1}^{(K-1) / 2} \frac{\zeta+\mathrm{e}^{-\zeta T}(2 i \pi \sin 2 i \pi T-\zeta \cos 2 i \pi T)}{\zeta^{2}+4 \pi^{2} i^{2}}
$$

Hence, $\widehat{X}_{\mathrm{e}}(\zeta, T):=\int_{0}^{T} \mathrm{~d} \tau \mathrm{e}^{-\zeta \tau} x(\tau)$ is given by

$$
\widehat{X}_{\mathrm{e}}(\zeta, T)=T_{0} \sum_{i=0}^{K-1} x_{i} \mathrm{e}^{-\zeta i / K}\left\{E_{K}\left(\frac{T}{T_{0}}-\frac{i}{K}\right)-E_{K}\left(-\frac{i}{K}\right)\right\} .
$$

\subsection{Calculation of $a(\tau), b(\tau)$}

Before we can compute $w_{11}(\tau)$, we need to find the unperturbed limit cycle, its period, $T_{0}$, the periodic function $\tilde{F}(\tau)$ and the mean of $F(\tau), f_{0}$. These are all straightforward: we solve the unperturbed equation (2.5) numerically as described in Section 4.1, obtaining $U_{0}, T_{0}$ and the solution and its derivative over one period. Since $u_{0}(\tau)$ is periodic, so is $h\left(u_{0}(\tau)\right)$, and so we can use equation (4.5) to estimate $F(\tau)$ for any $\tau$. From $F(\tau)$ we can then obtain $f_{0}$, and hence $\tilde{F}(\tau)$.

Computation of $w_{11}(\tau)$ can now be carried out from equation (2.15), but is complicated by the fact that, for $\tau=i T_{0} / 2, i \in \mathbb{Z}$, the integrand is singular and numerical integration techniques will break down. Singularity in the integrand, which is periodic, also prevents us from using equation (4.5). To discuss this further, let us define two subsets of $\mathbb{R}$ as $S=\cup_{i \in \mathbb{Z}} s_{i}$ with $s_{i}=$ $\left[i T_{0} / 2-r_{c}, i T_{0} / 2+r_{c}\right]$, where $r_{c} \ll T_{0} / 2$ is small and will be defined later; and $I=\mathbb{R} \backslash S$. We will then use a power series representation for $w_{11}(\tau), \tau \in S$, where the power series converges 'usefully' (the error term is less than the maximum acceptable error) for $|\tau| \leq r_{c}$, with Romberg integration [19], a standard numerical integration technique, being used for $\tau \in I$.

It should be pointed out here that we do not need to compute $\bar{\tau}$ explicitly. Instead, we can set the lower limit of the integral to any convenient value, $\tau_{l}$ say, provided we add a suitable multiple of $\dot{u}_{0}(\tau)$; so our definition becomes

$$
w_{11}(\tau)=\dot{u}_{0}(\tau) k_{2}+\dot{u}_{0}(\tau) \int_{\tau_{l}}^{\tau} d \tau^{\prime} c_{1} \frac{\mathrm{e}^{-F\left(\tau^{\prime}\right)}}{\dot{u}_{0}^{2}\left(\tau^{\prime}\right)}
$$

where the constant $k_{2}$, which depends on $\tau_{l}$, will be chosen to ensure continuity.

In practice, we only need to know $w_{11}(\tau)$ over a length of time consisting of two periods of $u_{0}(\tau)$, and so we calculate it for $\tau \in\left[0,2 T_{0}\right]$ : from Appendix $\mathrm{A}$ in [4, we know that $w_{11}(\tau)$ is well-defined even at $\tau=0$, which we take to be our value of $\tau_{l}$.

We derive the formal power series for $w_{11}(\tau)$ by using the method of Frobenius to solve the differential equation (2.5), with initial conditions chosen so as to ensure that the solution is on the limit cycle. Thus, $u_{0}(0)=U_{0}, \dot{u}_{0}(0)=0$, from which we obtain the power series in $\tau$ for $u_{0}(\tau)$ and hence, using term-by-term differentiation, for $\dot{u}_{0}(\tau)$. The latter is

$$
\dot{u}_{0}(\tau)=U_{0}\left(\alpha-\beta+\beta U_{0}^{2}\right)\left[-\tau+\left(1-\beta+3 \beta U_{0}^{2}\right) \frac{\tau^{2}}{2}\right]+O\left(\tau^{3}\right) .
$$


By Remark 1 in [4], $c_{1}=-r_{1}$, which is the coefficient of $\tau$ in the above series for $\dot{u}_{0}(\tau)$, and so $c_{1}=U_{0}\left(\alpha-\beta+\beta U_{0}^{2}\right)$. Using the series for $u_{0}(\tau)=U_{0}+\int_{0}^{\tau} d \tau^{\prime} \dot{u}_{0}\left(\tau^{\prime}\right)$, and term-by-term integration, we can also find power series for $F(\tau), \mathrm{e}^{-F(\tau)}$ and hence for the integrand $\mathrm{e}^{-F(\tau)} / \dot{u}_{0}^{2}(\tau)$. Integrating this series from 0 to $\tau$ term-by-term, and multiplying by the series for $c_{1} \dot{u}_{0}(\tau)$, we obtain $w_{11}(\tau)=1-\left(1-\beta+3 \beta U_{0}^{2}\right) \tau / 2+\left(1-2 \alpha+\beta^{2}\left(1-3 U_{0}^{2}\right)^{2}\right) \tau^{2} / 4+O\left(\tau^{3}\right)$. Finally, we apply the remaining condition on $\dot{w}_{11}$, that is, $\dot{w}_{11}(0)=0$, which forces the choice of $k_{2}$ in equation (4.8) to be such that $-k_{2} U_{0}\left(\alpha-\beta+\beta U_{0}^{2}\right)-\left(1-\beta+3 \beta U_{0}^{2}\right) / 2=0$. This gives

$$
w_{11}^{\mathrm{ser}}(\tau) \approx 1+\sum_{j=2}^{M} R_{j} \tau^{j}+O\left(\tau^{M+1}\right),
$$

where $2 R_{2}=\alpha-\beta+3 \beta u_{0}^{2}, 6 R_{3}=\alpha-\beta(1+\alpha-\beta)+3 \beta(1+3 \alpha-4 \beta) u_{0}^{2}+15 \beta^{2} u_{0}^{4}$ and so on. This is a truncation of the series actually used for $\tau \in s_{0}$. Using computer algebra, it is possible to extend this series to at least the term of order $\tau^{10}$, expressing each coefficient of $\tau$ as a polynomial in $\alpha, \beta$ and $U_{0}$ — that is, without assuming numerical values for these parameters - although the higher order coefficients become quite complicated.

The series (4.9) can be used to estimate $w_{11}(\tau)$ for $\tau \in s_{j}, j>0$, provided (i) the value given by the series is multiplied by $(-1)^{j} \mathrm{e}^{-j f_{0} T_{0} / 2}$ and (ii) $k_{2}$ is chosen so as to ensure continuity across the boundary of $s_{j}$. The term $(-1)^{j}$ in the correction factor arises as a result of the property of $u_{0}(\tau)$ in equation (2.6), and the exponential factor comes from the definition of $w_{11}(\tau)$, equation (2.15). Hence, $w_{11}(\tau)$ is estimated as

$$
w_{11}(\tau)=k_{2} \dot{u}_{0}(\tau)+(-1)^{j} \mathrm{e}^{-j f_{0} T_{0} / 2} w_{11}^{\mathrm{ser}}\left(\tau-j T_{0} / 2\right)+O\left(\tau^{M+1}\right)
$$

for $\tau \in s_{j}$.

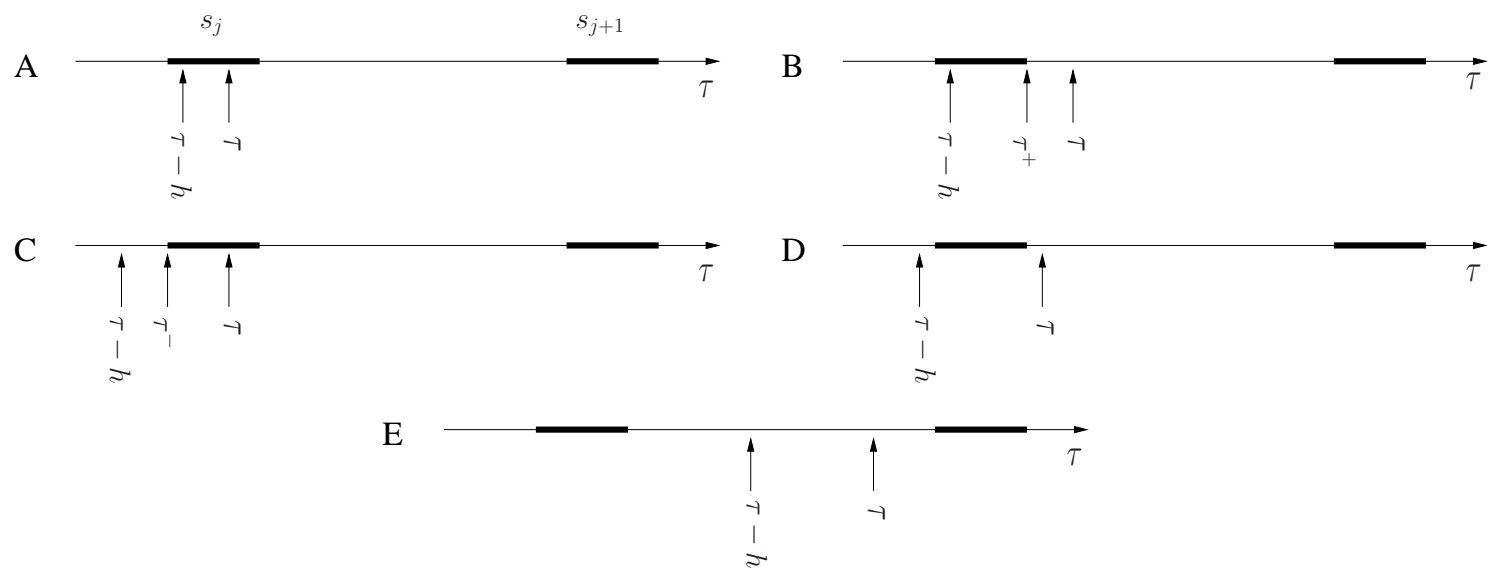

Figure 2: The cases A-E to be considered when calculating $w_{11}(\tau)$. The subsets $s_{j}=\left[j T_{0} / 2-r_{c}, j T_{0} / 2+r_{c}\right]$ and $s_{j+1}$ are shown as thick line segments. It is assumed that $w_{11}$ is being computed at equally spaced time steps of width $h$, that $w_{11}(\tau-h)$ has just been calculated, and that $w_{11}(\tau)$ is now to be found. How this is done depends on the relationship of $\tau$ to $\tau-h$ - see text.

In order to compute $b(\tau)$, we need to know $w_{11}(\tau)$ for $\tau \in\left[0,2 T_{0}\right]$ - see equation (4.11) - and hence we calculate $w_{11}(\tau)$ at equally spaced points $0, h, 2 h, \ldots, 2 T_{0}$, in that order, where $2 T_{0} / h$ is an integer. The point $\tau=0$ is in $s_{0}$ and so the truncated series is used here (with $k_{2}=0$ ). For $\tau>0$, various different cases exist, and these are illustrated in figure 2, in which $\tau$ is the time at which $w_{11}$ is to be calculated, and it is assumed that it has already been calculated at $\tau-h$.

- In case $\mathrm{A}, \tau \in s_{j}$, so the series is used, with the current value of $k_{2}$. 
- In case B, first $w_{11}\left(\tau^{+}\right) / \dot{u}_{0}\left(\tau^{+}\right)$, where $\tau^{+}=j T_{0} / 2+r_{c}$, is calculated from the truncated series. To this is added a numerical estimate of $\int_{\tau^{+}}^{\tau} \mathrm{d} \tau^{\prime} c_{1} \mathrm{e}^{-F\left(\tau^{\prime}\right)} / \dot{u}_{0}^{2}\left(\tau^{\prime}\right)$, and the result multiplied by $\dot{u}_{0}(\tau)$ to obtain an estimate of $w_{11}(\tau)$.

- In case $\mathrm{C}$, roughly the reverse happens. Define $\tau^{-}=j T_{0} / 2-r_{c}$. Then numerical integration is used to estimate $w_{11}\left(\tau^{-}\right)$, from which $k_{2}$ can be found, by assuming continuity across the boundary $\tau=\tau^{-}$. Since the appropriate value of $k_{2}$ is now known, the truncated series can be used to estimate $w_{11}(\tau)$.

- In case $\mathrm{D}$, compute as in $\mathrm{C}$, followed by B.

- In case E, straightforward numerical integration alone is used.

In this way, $w_{11}(i h)$ is computed for $i=0,1,2, \ldots, 2 T_{0} / h$, and it is now a simple matter to extract $a(\tau)$ and $b(\tau)$ at the points $\tau=i h, i=0,1, \ldots, T_{0} / h$, so that their values at any $\tau$ can be found by interpolation. From equation (2.18), we have that $w_{11}(\tau)=a(\tau)+\mathrm{e}^{-f_{0} \tau} b(\tau)$ and $a(\tau)=\gamma \dot{u}_{0}(\tau)$. Since $a(\tau)$ and $b(\tau)$ both have period $T_{0}$, we have

$$
b(\tau)=\mathrm{e}^{f_{0} \tau} \frac{w_{11}(\tau)-w_{11}\left(\tau+T_{0}\right)}{1-\mathrm{e}^{-f_{0} T_{0}}}
$$

and, knowing $w_{11}(\tau)$ for $\tau \in\left[0,2 T_{0}\right]$, we can now compute $b(\tau)$ for $\tau \in\left[0, T_{0}\right]$. Having computed $b(\tau)$, we can use for instance the method of least squares to estimate the value of $\gamma$ : that is, we find the value of $\gamma, \hat{\gamma}$, that minimises

$$
\sum_{i=0}^{T_{0} / h}\left[w_{11}(i h)-\mathrm{e}^{-f_{0} i h} b(i h)-\hat{\gamma} \dot{u}_{0}(i h)\right]^{2},
$$

which is

$$
\hat{\gamma}=\frac{\sum \dot{u}_{0}(i h)\left[w_{11}(i h)-\mathrm{e}^{-f_{0} i h} b(i h)\right]}{\sum \dot{u}_{0}^{2}(i h)},
$$

where the sums go from $i=0$ to $T_{0} / h$. This completes the calculation of $a(\tau)$ and $b(\tau)$.

\subsection{Illustrative results}

Illustrative results are now given for the case $\alpha=5, \beta=4$ and $f(\tau)=\sin \tau$. All the computations were carried out using double precision arithmetic. For interpolation, $K=151$ equally spaced points were used; the series for $I_{K}(\delta)$ was used if $|\delta|<\varepsilon_{I}=10^{-4}$. In series (4.9), $M=10$. In the definition of $s_{j}, r_{c}=10^{-2}$, and the fractional accuracy chosen for Romberg integration was $10^{-12}$. With these parameters, and $\rho=2$, we find $T_{0} \approx 3.698939867513906, U_{0} \approx 0.979106186033891, f_{0} \approx$ 0.757499334158 and $\hat{\gamma}=-54.855909271256$. Having computed $a(\tau)$ and $b(\tau)$, we can then estimate $A$, first of all from equation (2.24), using Romberg integration: this gives $A=16.0813516305191$. Using equation (4.5) to carry out the integration, we obtain $A=16.0813516305189$. Furthermore, we have from equation (2.28) that $A=-r_{1} \rho \Omega_{0}=U_{0}\left(\alpha-\beta+\beta U_{0}^{2}\right) /\left(\rho \Omega_{0}\right)=16.0813516307791$. These estimates agree with each other to 11 significant figures, thereby verifying the numerical techniques used to obtain them.

The calculation of $B_{11} \ldots B_{32}$ and $D_{1}, D_{2}$, defined after equation (2.27), now follows straightforwardly from equations (2.25). The only point to note is that these integrals can be zero, which gives problems in the error control scheme used for numerical integration. To overcome this, the integration is done in two parts, from 0 to $2 \pi p z$ and from $2 \pi p z$ to $2 \pi p$, with $z$ approximately, but not exactly, one half. We then find, for the above parameters, that, when $p=1, D_{1}, D_{2} \approx 10^{-12}$. On the other hand, with $p=2$, we find $D_{1} \approx 8.11989 \times 10^{-2}$ and $D_{2} \approx-5.20174 \times 10^{-1}$; for $p=4$, $D_{1}=-3.79022 \times 10^{-2}, D_{2}=2.74434 \times 10^{-1}$. 


\section{$5 \quad$ Numerical results}

To extend our analytical results to large values of $\mu$ we compute a set of Arnol'd tongues numerically. We make several comparisons between the theoretical predictions and the computational results, which provide information about the computational accuracy and the range of validity of some theoretical estimates.

\subsection{Arnol'd tongues}

We computed the Arnol'd tongues of system (2.1) for the 15 strongest resonances using the algorithms from 21] with two types of forcing: Harmonic forcing with

$$
f(t)=\sin t
$$

as considered in [4], and the forcing function

$$
f(\tau)=\frac{\lambda^{2}-1}{\lambda} \sum_{k=1}^{\infty} \frac{\sin k \tau}{\lambda^{k}}=\frac{\left(\lambda^{2}-1\right) \sin \tau}{\lambda^{2}+1-2 \lambda \cos \tau}, \quad \lambda>1
$$

containing all harmonics. Note that $f$ in (5.2) is smooth and $f(\tau) \in[-1,1]$; hence, it can be used as a direct replacement for the sine function in (5.1). The relative strength of the harmonics can be adjusted by varying $\lambda$, since one has $\hat{f}_{\nu}=\Phi(\lambda) \lambda^{-|\nu|}$, with $\Phi(\lambda)=\left(\lambda^{2}-1\right) / 2 \mathrm{i} \lambda$.

The results of both computations for the parameter values $\alpha=5$ and $\beta=4$ are illustrated and compared in figure 3. Note that in this case we have $\Omega_{0}=1.698645 \ldots$ and, hence, $\omega_{\mathrm{c}}(2)=$ $3.397290 \ldots$ and $\omega_{\mathrm{c}}(4)=6.794580 \ldots$ As explained in detail in 21 these tongues are computed by continuation of so-called constant- $\mu$ cross sections, starting at the tips. To facilitate our subsequent computations of the order of contact and opening angles we started with an extremely small continuation step size to obtain a large number of points very close to the tips for later fitting. For each tongue we performed 150 continuation steps. The computation of most tongues terminated by either reaching the computational boundary $\mu=3.5$ or by exceeding the maximal number of 150 continuation steps. However, the computation of some tongues, most notably of the 2:1 tongue, seems to end due to limitations of the algorithm we use; see [21] for more details. We did not pursue a further investigation, because we are mainly interested in the size and location of the 2:1 and 4:1 tongues for moderate $\mu$ and in investigating the behaviour at the tips of all tongues, for which we obtained sufficient data.

The bifurcation diagrams shown in figure 3 are clearly dominated by the strongest resonances occurring for $\rho=2$ and $\rho=4$. A continuation of the frequency-locked sub-harmonic solutions along the centre-lines $\omega / \Omega_{0}=2$ and $\omega / \Omega_{0}=4$ inside these tongues revealed that these solutions remain attracting for $\mu \leq 3.5$ at least along these centre-lines. On the other hand, we observe at the left-hand boundary of the 2:1 tongue that this tongue overlaps with other tongues. Hence, in these overlapping regions we might find multi-stability. To the right-hand side of the 2:1 tongue no such phenomenon is apparent in these figures.

The small plots to the right of figures 3 (a) and (b) show enlargements of the tip of the 3:1 tongue illustrating the effect of forcing with and without all harmonics present as predicted in Section 3.1 In figure 3 (a) we observe a high-order (quadratic) contact of the boundaries of the tongue, while in figure 3 (b) the two boundaries intersect transversally. Note that the slight shift to the left of $\omega / \Omega_{0}=3$ is due to the discretisation error of the periodic solutions; see 21] for technical details. It is remarkable, however, that our computations accurately capture the predicted highorder behaviour despite this approximation error, which is orders of magnitudes larger than the width of most tongues at their tips.

To quantify our findings and for comparison with the analytical predictions we developed a simple adaptive non-linear fitting algorithm for the width-function $\Delta \omega(\rho)$ to a monomial $\Delta \omega(\rho)=$ 

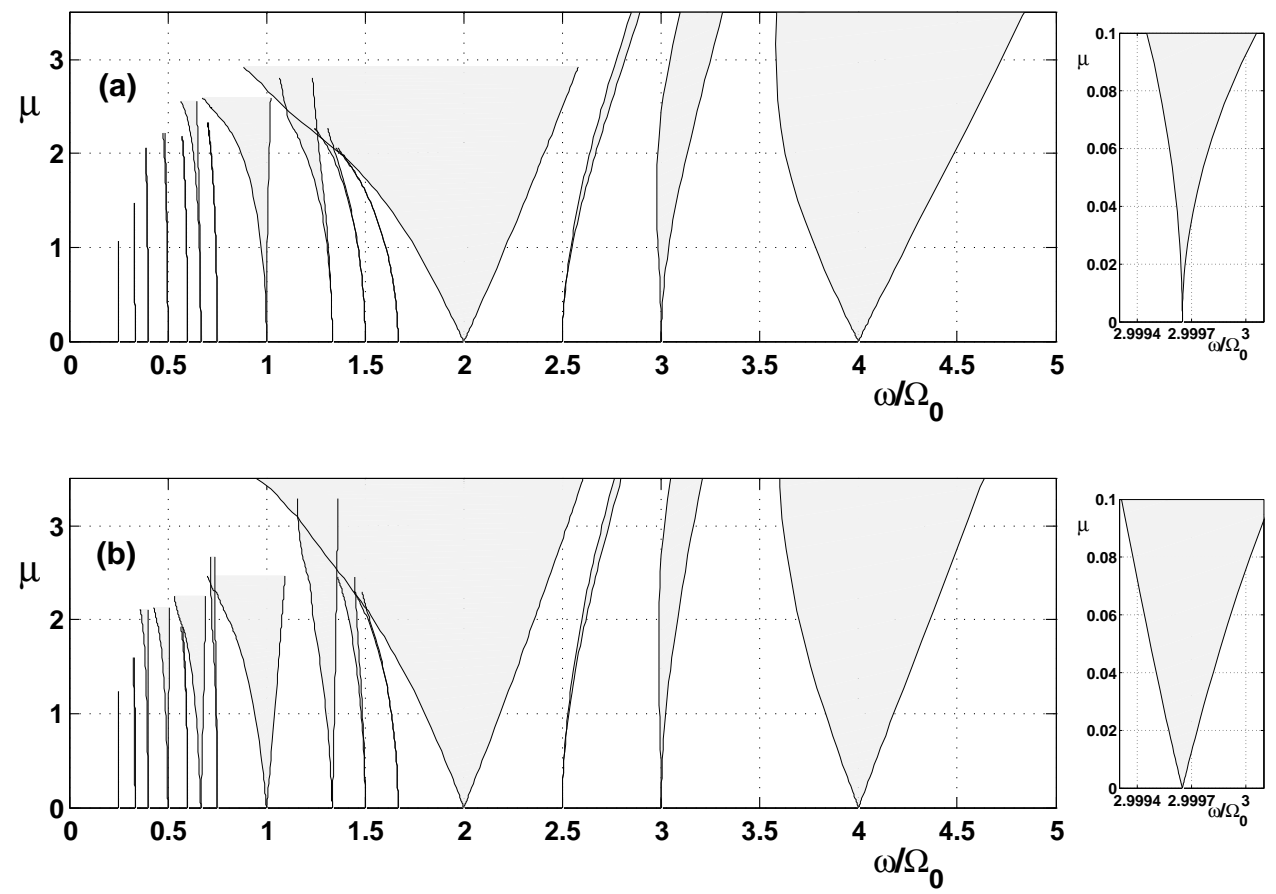

Figure 3: Some Arnol'd tongues in the $\left(\omega / \Omega_{0}, \mu\right)$-plane for the ILFD for $\alpha=5$ and $\beta=4$ with (a) $f(\tau)=\sin \tau$ and (b) $f(\tau)$ given by (5.2) with $\lambda=2$. For these parameter values we have $\Omega_{0}=1.69864489$, $T_{0}=2 \pi / \Omega_{0}=3.69893987, A \rho=2.78668166, D_{1}=0.00703534$ and $D_{2}=-0.0450695$.

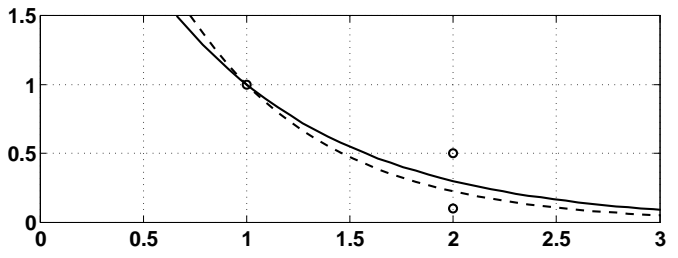

(a)

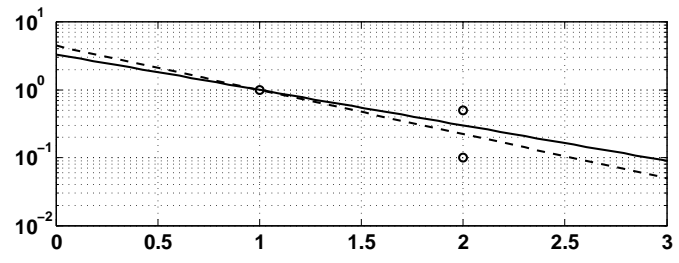

(b)

Figure 4: Comparison of linear (dashed) and non-linear (solid) fits for a simple test example.

$a \mu^{b}$ with $a$ and $b$ unknown. One might argue that one could use a linear fit to logarithmic data of the form $\ln (\Delta \omega(\rho))=\ln a+b \ln \mu$ to compute estimates for $a$ and $b$. However, this leads to biased estimates as we illustrate in figure 4 where we compare the results of a non-linear fit (solid) with a linear fit (dashed) to the function $y=a \exp (b x)$. Only the non-linear fit is a useful fit to the data as figure 4 (a) clearly illustrates, the linear fit here being biased towards lower function values. There are several reasons why a linear fit to logarithmic data is inappropriate, the most important ones being that the two least-squares residual functions $\|Y-a \exp (b X)\|_{2}^{2}$ and $\|\ln Y-(\ln a+b X)\|_{2}^{2}$ have different minimisers, and that $Y$ and $\ln Y$ do not have the same error distribution. Another suggestion could be to compute a linear fit to a polynomial $p_{n}(\mu)=a_{0}+a_{1} \mu+\cdots+a_{n} \mu^{n}$ of sufficiently high order $n$. However, we found that this leads to a least-squares problem that is so ill-conditioned that round-off errors become amplified to order one, that is, the fitted coefficients are essentially meaningless. A way out is to use orthonormal polynomials as base functions instead of monomials of the form $a_{k} \mu^{k}$. However, since our non-linear fit worked sufficiently well we did not pursue this further. 


\begin{tabular}{|c|c|c|c|c|c|c|}
\hline \multirow[b]{2}{*}{$p: q$} & \multicolumn{3}{|c|}{$f(\tau)=\sin (\tau)$} & \multicolumn{3}{|c|}{$f(\tau)=\frac{\left(\lambda^{2}-1\right) \sin \tau}{\lambda^{2}+1-2 \lambda \cos \tau}, \lambda=2$} \\
\hline & $\Delta \omega(p / q)$ & $\mu_{\mathrm{fit}}$ & $N_{\text {fit }}$ & $\Delta \omega(p / q)$ & $\mu_{\text {fit }}$ & $N_{\text {fit }}$ \\
\hline $1: 4$ & $2.770 \times 10^{-7} \mu^{8.076}$ & $8.763 \times 10^{-1}$ & 48 & $5.571 \times 10^{-4} \mu^{1.001}$ & $4.481 \times 10^{-3}$ & 72 \\
\hline $1: 3$ & $1.055 \times 10^{-5} \mu^{6.052}$ & $8.971 \times 10^{-1}$ & 40 & $2.971 \times 10^{-3} \mu^{1.001}$ & $5.932 \times 10^{-3}$ & 73 \\
\hline $2: 5$ & $7.554 \times 10^{-5} \mu^{5.038}$ & $8.294 \times 10^{-1}$ & 36 & $7.128 \times 10^{-3} \mu^{1.001}$ & $7.913 \times 10^{-3}$ & 73 \\
\hline $1: 2$ & $5.188 \times 10^{-4} \mu^{4.014}$ & $6.529 \times 10^{-1}$ & 40 & $1.786 \times 10^{-2} \mu^{1.001}$ & $1.068 \times 10^{-2}$ & 74 \\
\hline $3: 5$ & $3.093 \times 10^{-8} \mu^{10.19}$ & $8.839 \times 10^{-1}$ & 14 & $1.057 \times 10^{-5} \mu^{1.001}$ & $7.612 \times 10^{-4}$ & 58 \\
\hline $2: 3$ & $4.096 \times 10^{-3} \mu^{3.003}$ & $2.662 \times 10^{-1}$ & 49 & $4.780 \times 10^{-2} \mu^{1.001}$ & $1.612 \times 10^{-2}$ & 74 \\
\hline $3: 4$ & $2.409 \times 10^{-6} \mu^{8.016}$ & $7.462 \times 10^{-1}$ & 16 & $5.101 \times 10^{-5} \mu^{0.9990}$ & $7.016 \times 10^{-4}$ & 55 \\
\hline $1: 1$ & $4.828 \times 10^{-2} \mu^{2.000}$ & $9.099 \times 10^{-2}$ & 76 & $1.467 \times 10^{-1} \mu^{1.001}$ & $3.179 \times 10^{-2}$ & 76 \\
\hline $4: 3$ & $9.979 \times 10^{-3} \mu^{3.001}$ & $1.540 \times 10^{-1}$ & 47 & $5.016 \times 10^{-2} \mu^{1.001}$ & $1.097 \times 10^{-2}$ & 70 \\
\hline $3: 2$ & $1.112 \times 10^{-3} \mu^{4.014}$ & $5.338 \times 10^{-1}$ & 38 & $1.688 \times 10^{-3} \mu^{1.001}$ & $1.973 \times 10^{-3}$ & 61 \\
\hline $5: 3$ & $5.049 \times 10^{-5} \mu^{5.971}$ & $2.588 \times 10^{-1}$ & 16 & $2.854 \times 10^{-5} \mu^{0.9990}$ & $5.950 \times 10^{-4}$ & 55 \\
\hline $2: 1$ & $7.556 \times 10^{-1} \mu^{1.000}$ & $9.684 \times 10^{-2}$ & 80 & $6.280 \times 10^{-1} \mu^{1.000}$ & $9.024 \times 10^{-2}$ & 79 \\
\hline $5: 2$ & $1.595 \times 10^{-2} \mu^{3.971}$ & $2.039 \times 10^{-1}$ & 35 & $1.832 \times 10^{-4} \mu^{0.9990}$ & $4.539 \times 10^{-4}$ & 50 \\
\hline $3: 1$ & $1.024 \times 10^{-1} \mu^{1.999}$ & $8.960 \times 10^{-2}$ & 77 & $1.331 \times 10^{-2} \mu^{0.9994}$ & $2.410 \times 10^{-2}$ & 73 \\
\hline $4: 1$ & $7.957 \times 10^{-1} \mu^{0.9999}$ & $9.719 \times 10^{-2}$ & 80 & $5.968 \times 10^{-1} \mu^{0.9999}$ & $9.757 \times 10^{-2}$ & 79 \\
\hline
\end{tabular}

Table 1: Leading contributions to the plateau widths corresponding to the main resonances as they appear in figure 3 from left to right. These coefficients were obtained by fitting the monomial $a \mu^{b}$ to the numerically computed values for $\Delta \omega(p / q)$ over the interval $\mu \in\left[0, \mu_{\mathrm{fit}}\right]$ on $N_{\text {fit }}$ data points.

For our computations we used the weighted least-squares residual function

$$
F(a, b):=\left\|W(\mu)\left(\Delta \omega(\rho)-a \mu^{b}\right)\right\|_{2}^{2},
$$

where we used the weight-function $W(\mu)=1 / \mu$ to penalise errors closer to the tip $\mu=0$. The numerical data is normalised to the unit square, that is, we compute a fit to $\Delta \omega(\rho) / \max \{\Delta \omega(\rho)\}$ and $\mu / \max \{\mu\}$. We computed estimates for $a$ and $b$ by applying Newton's method to the equations $\partial F(a, b) / \partial(a, b)=0$ and rescaled the computed coefficients to fit the original data. The size of the fitting interval $\mu \in\left[0, \mu_{\mathrm{fit}}\right]$ was computed adaptively. We started with an initial fitting interval $\mu_{\mathrm{fit}}=\max \left\{0.1, \operatorname{argmax}\left\{\Delta \omega(\rho)<10^{-4}\right\}\right\}$, that is, we used either $\mu_{\mathrm{fit}}=0.1$ or the largest value of $\mu$ such that the width of the tongue was less than $10^{-4}$. The fitting interval was accepted if the least squares error $F(a, b)$ for the normalised data was less than $10^{-3}$ and reduced successively if the error was larger. Within the fitting interval we excluded points for which $\Delta \omega(\rho)$ was zero within numerical accuracy. Table 1 summarises our results for both forms of forcing. Each row states the fitted monomial representing the leading order term together with the fitting interval and the number $N_{\text {fit }}$ of data points this monomial was fitted to. These computations agree extremely well with the theoretical predictions and also verify that our fitting algorithm is suitable to capture the leading-order behaviour accurately.

First of all, our computations of the width of the locking intervals are in alignment with our theoretical results

$$
\Delta \omega(p / q)= \begin{cases}O\left(\mu^{k}\right) & \text { for } p \text { even, } \\ O\left(\mu^{2 k}\right) & \text { for } p \text { odd }\end{cases}
$$

for harmonic forcing (5.1), and with

$$
\Delta \omega(p / q)=O(\mu)
$$

for general forcing (5.2) containing all harmonics. Furthermore, for the main Arnol'd tongues corresponding to the resonances 2:1 and 4:1, equation (2.27) valid for harmonic forcing reduces to

$$
\mathfrak{D}_{1}\left(\tau_{0}\right)=\frac{1}{A}\left(D_{1} \cos \tau_{0}+D_{2} \sin \tau_{0}\right),
$$


where, for the 2:1 resonance, $A=16.0814, D_{1}=8.11989 \times 10^{-2}$, and $D_{2}=-5.20174 \times 10^{-1}$; see also Section 4.4. An easy computation gives

$$
\max _{0 \leq \tau_{0} \leq 2 \pi} \mathfrak{D}_{1}\left(\tau_{0}\right)=-\min _{0 \leq \tau_{0} \leq 2 \pi} \mathfrak{D}_{1}\left(\tau_{0}\right)=M, \quad M=0.0327381 .
$$

For example, for the boundaries of the tongue corresponding to the 2:1 resonance we find from table 1 that $\left(\tan \theta_{1}(2)+\tan \theta_{2}(2)\right) / 2 \approx 0.7556 / 2=0.3778$ in agreement with (2.32), which gives $\rho^{2} \Omega_{0}^{2} M=0.37785$. Similarly, for the $4: 1$ resonance we have $A=32.1627, D_{1}=-3.79022 \times 10^{-2}$, $D_{2}=2.74434 \times 10^{-1}$, giving $M=8.6137 \times 10^{-3}$. We compute that $\rho^{2} \Omega_{0}^{2} M=0.39766$, again in agreement with the result in table 1 that $\left(\tan \theta_{1}(4)+\tan \theta_{2}(4)\right) / 2=0.7959 / 2=0.39795$.

Also for the secondary resonances $p: 1$, with $p$ odd, the agreement between the numerical results and the analytical predictions is satisfactory. The second order computation, performed according to the analysis in Section 3.2. gives, for the $1: 1$ and $3: 1$ resonances, the values $\Delta(1) \approx 4.8246 \times 10^{-2}$ and $\Delta(3) \approx 1.0269 \times 10^{-1}$, to be compared with the values $4.828 \times 10^{-2}$ and $1.024 \times 10^{-1}$ in table 1

For the all-harmonics forcing (5.2), the plateau widths as given in table 1 are consistent with the scaling law (3.10). If we fix $p$ to be an odd integer, then $\nu_{0} \rho=\nu_{0} p / q=2 p$, hence $\nu_{0}=2 q$, and we find $\left|\hat{f}_{\nu_{0}}\right|\left|\bar{K}_{\nu_{0} \rho}(\rho)\right|=\Phi(\lambda) \lambda^{-2 q}\left|\bar{K}_{2 p}(p / q)\right|$. If, on the contrary, we fix $p$ to be an even integer then $\nu_{0} \rho=\nu_{0} p / q=p$, hence $\nu_{0}=q$, so that we obtain $\left|\hat{f}_{\nu_{0}}\right|\left|\bar{K}_{\nu_{0} \rho}(\rho)\right|=\Phi(\lambda) \lambda^{-q}\left|\bar{K}_{p}(p / q)\right|$. When inserted into (3.10), this leads to

$$
|\Delta \omega(\rho)| \approx \begin{cases}c \mu /\left(q 2^{2 q}\right) & \text { for } p \text { odd } \\ c \mu /\left(q 2^{q}\right) & \text { for } p \text { even }\end{cases}
$$

with the constant $c=c(p)$ independent of $q$. The constant $c$ can be computed using the theory. A comparison between (5.5) and (3.10) gives $c(p)=\ln 2 \Omega_{0}\left|\bar{r}_{1}\right|^{-1} \Phi(\lambda) p\left|\bar{K}_{\nu_{0} \rho}(p / q)\right|$, with $\nu_{0} \rho=2 q$ for odd $p$ and $\nu_{0} \rho=q$ for even $p$. For $p=1,2,3$ we compute $c(p)=0.82,1.64,0.11$, respectively. These estimates are consistent with our numerical data in table 1. Fitting our data to function (5.5) we obtain the numerical estimates $c(p)=0.5867,1.255,0.05326$, respectively, which is in good agreement considering the limited numerical accuracy and that (5.5) is valid for $q \rightarrow \infty$. Figure 5 shows a comparison between the theoretically and numerically obtained width functions.

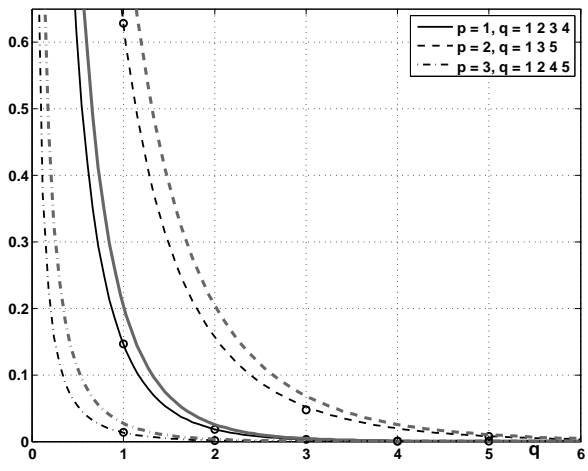

(a)

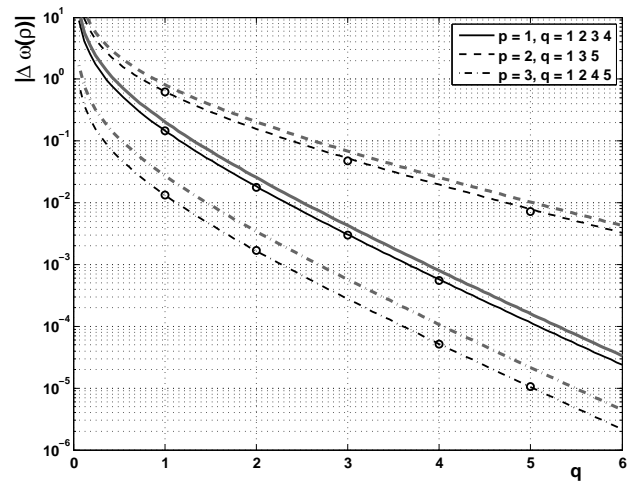

(b)

Figure 5: Tongue widths $|\Delta \omega(\rho)|$ for fixed $p$ and varying $q$. The black curves are plots of (5.5) using the constants $c$ from the numerical data and the grey curves are plots of (5.5) using the constants $c$ predicted by the theory. The discrepancies are due to the small values of $q$ are are expected to be asymptotically zero for large $q$. 


\subsection{Width of plateaux as a function of $\alpha$ and $\beta$}

Of practical importance is the rate at which the width of a given locking intereval increases with increasing $\mu$. The locking regions, i.e. the Arnol'd tongues, are cone-shaped and the vertical angle of the cone, $2 \theta_{1}(\rho)$, which is a measure of width growth rate, depends on the parameters $\alpha$ and $\beta$. This angle can be computed from equation (2.36) for a given $\rho$, and typical results are given in figure 6 for $\rho=2$ and a variety of values of $\beta$, with $\alpha \in(\beta, 10]$.

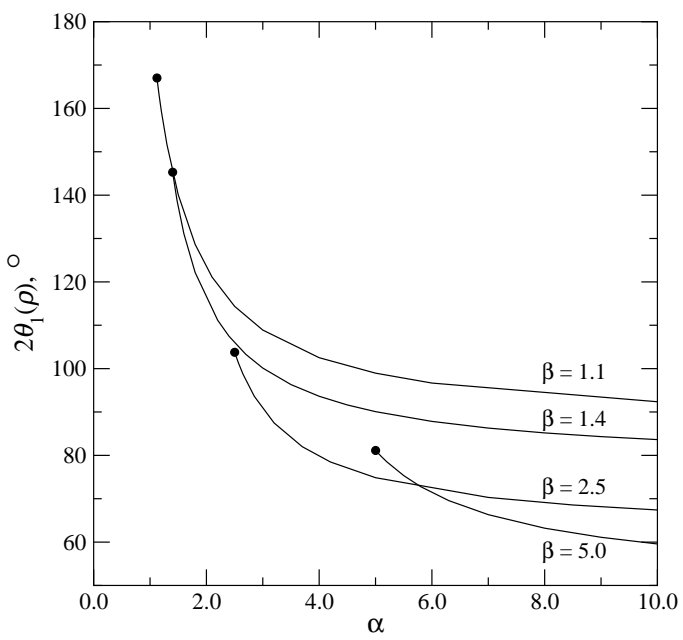

Figure 6: A plot of $2 \theta_{1}(2)$, the opening angle (degrees) of the Arnol'd tongue for $\rho=2$, against $\alpha$, for various values of $\beta$, with $\alpha>\beta>1$. The angles have been computed numerically from equation (2.36).

\section{Conclusions}

In this paper we have investigated both analytically and numerically the structure of the Arnol'd tongues for a resonant injection-locked frequency divider (ILFD). It is the natural extension of the analysis performed in [4, where we analytically proved the experimental and numerical results contained in [15, 16] by providing explicit formulae for the width of the plateaux appearing in the devil's staircase. More precisely in 4 we found the following result. Denote by $\omega$ and $\Omega$ the frequencies of the driving signal and of the output signal of the ILFD, respectively, with $\mu$ the driving amplitude. Then, if for $\rho \in \mathbb{Q}$ we call $\Delta \omega(\rho)=\{\omega: \omega / \Omega=\rho\}$ the width of the corresponding locking interval, we showed that $\Delta \omega(\rho)$ satisfies $\Delta \omega(2 n / k)=O\left(\mu^{k}\right)$ and $\Delta \omega((2 n-$ $1) / k)=O\left(\mu^{2 k}\right)$ for all $k, n \in \mathbb{N}$ such that $2 n / k$ and $(2 n-1) / k$ are irreducible fractions. In particular this implies that the largest plateaux correspond to even integer values of the ratio $\omega / \Omega$.

In this paper we have extended the above results: we studied the system of ordinary differential equations (2.1), (2.2), (2.3), which describe the ILFD, with a more general driving term in the form of any analytic periodic function (we confined ourselves to functions containing odd-harmonics only in order to make the analysis more transparent and yet without any significant loss of generality). In [4] we used $f(t)=\sin (t)$ (one harmonic only), as in [15, 16]. Here, we studied the locking intervals $\Delta \omega(\rho)$ by using in (2.3) a $2 \pi$-periodic function of the form $f(t)=\sum_{\nu=1}^{\infty} \hat{f}_{\nu} \sin \nu t$, with $\left|\hat{f}_{\nu}\right| \leq \Phi \mathrm{e}^{-\xi|\nu|}$ (by analyticity). We found that, for any $\rho=p / q \in \mathbb{Q}$, with $p, q$ relatively prime integers, the key condition for the existence of the locking $\omega=\rho \Omega$ (and hence of a plateau), is that there exists $\nu$ such that $\hat{f}_{\nu} \neq 0$ and $2\left|\nu^{\prime}\right| q=|\nu| p$ and some $\nu^{\prime} \in \mathbb{Z}$. This condition is certainly satisfied if, for instance, $\left|\nu^{\prime}\right|=p$ and $|\nu|=2 q$, provided $\hat{f}_{2 q} \neq 0$, or $\left|\nu^{\prime}\right|=2 p$ and $|\nu|=q$, provided $\hat{f}_{q} \neq 0$. Thus, for any resonance $p: q$ one has a plateau $\Delta \omega(p / q)$ which to first order is given by 
(3.1) and (3.6). In particular, to leading order, the width of the Arnol'd tongues is expressed as $\Delta \omega_{1}(\rho) \approx 2 \mu \rho \Omega_{0}\left|\bar{r}_{1}\right|^{-1}\left|\hat{f}_{\nu_{0}}\right|\left|\bar{K}_{\nu_{0} \rho}(\rho)\right|$, where $\rho=p / q, \Omega_{0}$ and $\bar{r}_{1}$ are constants depending on the unperturbed system (but not on the driving) and $\nu_{0} \geq 1$ denotes the integer which provides the leading coefficient in the sum (3.6). Note that the formula reduces to the one obtained in 4 - as it should - if $f(t)=\sin (t)$ : in that case $\hat{f}_{\nu} \neq 0$ only for $\nu=1$, so that $q=1$ and $p \in 2 \mathbb{N}$.

Moreover, by keeping in (3.6) the whole sum, we obtain $|\Delta \omega(\rho)| \leq \mu C p^{2} q^{-1} \mathrm{e}^{-\xi_{1} p} \mathrm{e}^{-\xi q}$ where $C$ is a constant independent of $p$ and $q$, thereby showing that the Arnol'd tongues have width proportional to $\mu$, but with proportionality constants which decay exponentially with $p$ and $q$.

We have also computed analytically the contribution of the second order, namely the coefficient of $\mu^{2}$. In this case one needs to compute the first order solution $\left(u_{1}(\tau), \dot{u}_{1}(\tau)\right)$, with $u_{1}(\tau)$ given in (3.17), which rather complicates the analysis. We found $\Delta \omega(\rho)=\mu^{2} \Delta_{2} \omega(\rho)+O\left(\mu^{3}\right)$, which replaces (3.1) when the first order vanishes. For instance, if $f$ contains only the first harmonics then the condition for locking onto a $p: q$ resonance becomes: $2\left|\nu^{\prime}\right| q=\left|\nu_{1}+\nu_{2}\right| p$, with $\hat{f}_{\nu_{1}} \hat{f}_{\nu_{2}} \neq 0$, is satisfied for some $\nu^{\prime} \in \mathbb{Z}$. This shows that when $f(t)=\sin t$, as in 15, 16, 4, the plateaux corresponding to odd $\rho$ are of order $\mu^{2}$.

Higher order contributions can in principle be computed with a very similar strategy (see (3.23) and (3.24) ); the important point to notice is that to all order $k$ the coefficients $\Delta_{k} \omega(\rho)$ decay exponentially in both $p$ and $q$. Naturally higher order terms become dominant when all the terms of smaller order vanish.

To complete our investigation, we computed the functions $\mathfrak{D}_{1}\left(\tau_{0}\right)$ and $\mathfrak{D}_{2}\left(\tau_{0}\right)$ numerically, from which the tongue widths $\Delta \omega(\rho)$ and $\Delta \omega_{2}(\rho)$ can be calculated, via equations (3.1) and (3.22) respectively. Some of the techniques required to carry out this computation are described in Section 3 . We then computed a set of Arnol'd tongues, which was sufficiently large for testing the numerics on the basis of the theoretical predictions. In particular, we computed the width of the tongues for two types of forcing: (i) only one harmonic and (ii) all harmonics present in the Fourier expansion. Our computational results are in excellent alignment with the theory as stated above, which supports our belief that the locking charts in figure 3 are accurate. These two charts clearly demonstrate the dominance of the 2:1 and the 4:1 resonances. Furthermore, a comparison indicates that the location of the tongues is robust under generic perturbations; the differences in the shapes of the tongues are small.

\section{A Error in the interpolation scheme}

Starting from equation (4.1), we expand the sine functions in terms of complex exponentials to obtain

$$
I_{K}(t)=\frac{1}{K} \sum_{|j| \leq(K-1) / 2} \mathrm{e}^{2 \mathrm{i} j \pi t} .
$$

Setting $t=\tau / T_{0}$, so that the scheme can be used to interpolate a periodic function $x(t)$ of arbitrary period $T_{0}$ in terms of $\tau$, we have the Fourier expansion

$$
x(t)=\sum_{n \in \mathbb{Z}} \alpha_{n} \mathrm{e}^{2 \mathrm{i} n \pi t}
$$

We interpolate $x(t)$ by

$$
\hat{x}(t)=\sum_{j=0}^{K-1} x(j / K) I_{K}(t-j / K)=\sum_{|m| \leq(K-1) / 2} \beta_{m} \mathrm{e}^{2 \mathrm{i} m \pi t},
$$

where the last inequality follows from equation (A.1). In order to determine how well $x(t)$ is approximated by $\hat{x}(t)$, we need to compare $\alpha_{m}$ with $\beta_{m}$. Substituting for $x(j / K)$ and $I_{K}(t)$ in 
equation (A.2) and rearranging, we find

$$
\hat{x}(t)=\sum_{|m| \leq(K-1) / 2} \mathrm{e}^{2 \mathrm{i} m \pi t}\left\{\sum_{n \in \mathbb{Z}} \frac{\alpha_{n}}{K} \sum_{j=0}^{K-1} \mathrm{e}^{2 \mathrm{i}(n-m) j \pi / K}\right\},
$$

where the term in braces is equal to $\beta_{m}$. The sum over $j$ is equal to $\left[1-\mathrm{e}^{2 \mathrm{i}(n-m) \pi}\right] /\left[1-\mathrm{e}^{2 \mathrm{i}(n-m) \pi / K}\right]$ provided that $(n-m) \neq p K, p \in \mathbb{Z}$, and is equal to $K$ otherwise. Hence,

$$
\frac{1}{K} \sum_{j=0}^{K-1} \mathrm{e}^{2 \mathrm{i}(n-m) j \pi / K}= \begin{cases}0 & n-m \neq p K \\ 1 & n-m=p K .\end{cases}
$$

Hence

$$
\beta_{m}=\sum_{p \in \mathbb{Z}} \alpha_{m+p K}=\alpha_{m}+\alpha_{m-K}+\alpha_{m+K}+\ldots
$$

Now, since $x(t)$ is the solution of an ODE with analytic coefficients, it is itself analytic, and so, for all $n,\left|\alpha_{n}\right|<C_{1} \mathrm{e}^{-C_{2}|n|}$, where $C_{1}, C_{2}$ are positive real constants. Thus,

$$
\left|\beta_{m}-\alpha_{m}\right|<C_{1} \mathrm{e}^{-C_{2} K}
$$

and hence, by choosing $K$ sufficiently large, the interpolation error can be made as small as we please.

Acknowledgments. We thank Peter Kennedy for bringing this problem to our attention, and for the interest shown in our results. We are also indebted to Bill Christmas for useful discussions on interpolation. FS was supported by the Engineering and Physical Sciences Research Council (EPSRC) Grant no. EP/D063906/1.

\section{References}

[1] A.A. Abidi, L.O. Chua, On the dynamics of Josephson-junction circuits, Electron. Circuits Syst. 3 (1979), no. 4, 974-980.

[2] A. Amann, M.P. Mortell, E.P. O'Reilly, M. Quinlan, D. Rachinskii, Mechanisms of syncronization in frequency dividers, IEEE Trans. Circuits Syst. I. Regul. Pap. 56 (2009), no.1, 190-199.

[3] V.I. Arnol'd, Geometrical methods in the theory of ordinary differential equations, Grundlehren der Mathematischen Wissenschaften Vol. 250, Springer, New York, 1988.

[4] M. Bartuccelli, J. Deane, G. Gentile, Frequency locking in the injection-locked frequency divider equation, Proc. R. Soc. Lond. Ser. A Math. Phys. Eng. Sci. 465 (2009), no. 2101, 283-306.

[5] Y. F. Chang and G. Corliss, Ratio-like and recurrence relation tests for convergence of series, J. Inst. Math. Appl. 25 (1980), 349-359.

[6] W.A. Coppel, Some quadratic systems with at most one limit cycle, Dynamics reported Vol. 2, 61-88, Dynam. Report. Ser. Dynam. Systems Appl., 2, Wiley, Chichester, 1989.

[7] S. Daneshgar, O. De Feo, M.P. Kennedy, Obsrvations concerning the locking range in a complementary differential LC injection-locked frequency divider. Part I: qualitative analysis, IEEE Trans. Circuits Syst. I. Regul. Pap., to appear.

[8] G. Gentile, M. Bartuccelli, J. Deane, Bifurcation curves of subharmonic solutions and Melnikov theory under degeneracies, Rev. Math. Phys. 19 (2007), no. 3, 307-348. 
[9] J. Guckenheimer, Ph. Holmes, Nonlinear oscillations, dynamical systems, and bifurcations of vector fields, Applied Mathematical Sciences Vol. 42, Springer, New York, 1990.

[10] Ph. Hartman, Ordinary differential equations, Classics in Applied Mathematics Vol. 38, Society for Industrial and Applied Mathematics (SIAM), Philadelphia, PA, 2002.

[11] M.R. Herman, Mesure de Lebesgue et nombre de rotation, Geometry and topology (Proc. III Latin Amer. School of Math., Inst. Mat. Pura Aplicada CNPq, Rio de Janeiro, 1976), pp. 271-293, Lecture Notes in Mathematics, Vol 597, Springer, Berlin, 1977.

[12] M.P. Kennedy, L.O. Chua, Van der Pol and chaos, IEEE Trans. Circuits Syst. I. Regul. Pap. 33 (1986), no. 10, 974-980.

[13] M. Levi, Nonchaotic behavior in the Josephson junction, Phys. Rev. A (3) 37 (1988), no. 3, 927-931.

[14] P. Maffezzoni, Analysis of oscillator injection locking through phase-domain impulse-response, IEEE Trans. Circuits Syst. I. Regul. Pap. 55 (2008), no. 5, 1297-1305.

[15] D. O'Neill, D. Bourke, M.P. Kennedy, The Devil's staircase as a method of comparing injection-locked frequency divider topologies, Proceedings of the 2005 European Conference on Circuit Theory and Design, 2005, Vol. III, pp. 317-320.

[16] D. O'Neill, D. Bourke, Zh. Ye, M.P. Kennedy, Accurate modeling and experimental validation of an injection-locked frequency divider, Proceedings of the 2005 European Conference on Circuit Theory and Design, 2005, Vol. III, pp. 409-412.

[17] U. Parlitz, W. Lauterborn, Period-doubling cascades and devil's staircases of the driven van der Pol oscillator, Phys. Rev. A 36 (1987), no. 3, 1428 - 1434.

[18] L. Pivka, A.L. Zheleznyak, L.O. Chua, Arnol'd tongues, devil's staircase, and self-similarity in the driven Chua's circuit, Internat. J. Bifur. Chaos Appl. Sci. Engrg. 4 (1994), no. 6, 1743-1753.

[19] W.H. Press, S.A. Teukolsky, W.T. Vetterling, B.P. Flannery, Numerical Recipes in $C$ (2nd edition, 1992), Cambridge University Press, Cambridge.

[20] M. Qian, J.-Z. Wang, X.-J. Zhang, Resonant regions of Josephson junction equation in case of large damping, Phys. Lett. A 372 (2008), no. 20, 3640-3644.

[21] F. Schilder, B. B. Peckham, Computing Arnol'd tongue scenarios, J. Comput. Phys. 220 (2006) no. 2, 932-951.

[22] B. van der Pol, J. van der Mark, Frequency demultiplication, Nature 120 (1927), no. 3019, 363-364.

[23] Zh. Ye, T. Xu, M.P. Kennedy, Locking range analysis for injection-locked frequency dividers, Proceedings of the 2006 IEEE International Symposium on Circuits and Systems, 2006, 4070-4073.

[24] Zh. F. Zhang, Proof of the uniqueness theorem of limit cycles of generalized Liénard equations, Appl. Anal. 23 (1986), no. 1-2, 63-76. 